\title{
Non-Backtracking Loop Soups and Statistical Mechanics on Spin Networks
}

\author{
Federico Camia and Marcin Lis
}

\begin{abstract}
We introduce and study a Markov field on the edges of a graph $\mathcal{G}$ in dimension $d \geq 2$ whose configurations are spin networks. The field arises naturally as the edge-occupation field of a Poissonian model (a soup) of non-backtracking loops and walks characterized by a spatial Markov property such that, conditionally on the value of the edge-occupation field on a boundary set that splits the graph into two parts, the distributions of the loops and arcs contained in the two parts are independent of each other. The field has a Gibbs distribution with a Hamiltonian given by a sum of terms which involve only edges incident on the same vertex. Its free energy density and other quantities can be computed exactly, and their critical behavior analyzed, in any dimension.
\end{abstract}

\section{Introduction}

The free energy density is an important tool and one of the main objects of study in statistical mechanics, since thermodynamic functions can be expressed in terms of its derivatives. As a consequence, models whose free energy density can be computed exactly have played a crucial role in the development of statistical mechanics. Perhaps the main example is the two-dimensional Ising model whose free energy density was famously derived by Onsager. However, this situation is rare and typically restricted to dimensions one and two, as in the case of the Ising model.

In this paper, we introduce and study a Markov field whose free energy density can be computed exactly in any dimension. The field arises naturally as the edge-occupation field of a stochastic model of non-backtracking loops and walks, but it does not seem to have been studied before. Such random loop models first appeared in the work of Symanzik [31] on Euclidean field theory, and can be used to prove triviality (i.e., the Gaussian nature) of Euclidean 
fields in dimensions five and higher $[1,13]$. A lattice version appears in the work of Brydges, Fröhlich and Spencer [7], who developed a random walk representation of spin systems. The loops that appear in the work of Brydges, Fröhlich and Spencer are allowed to backtrack.

A prototypical example of a statistical mechanical model whose partition function coincides with that of a loop model is the discrete Gaussian free field; its partition function can be expressed as the grand-canonical partition function of an ideal lattice gas of loops, i.e., a Poissonian ensemble of lattice loops. This is a special case of the random walk representation of Brydges, Fröhlich and Spencer [7]. In this case, the obvious triviality of the Gaussian free field is reflected in the Poissonian nature of the ensemble of loops. In general, the ensembles of random paths and loops that appear in the work of Brydges, Fröhlich and Spencer are not Poissonian: the underlying Poisson distribution is "tilted" by means of a potential which is a function of the vertexoccupation field generated by the random loops and paths. This suggests that the occupation field of a Poissonian ensemble of loops is an interesting object to study. Such an occupation field is the main object of interest in this paper, although we look at edge occupation and our loops have a non-backtracking condition that is not present in the work of Brydges, Fröhlich and Spencer.

The Poissonian ensemble of loops implicit in the work of Symanzik was rediscovered by Lawler and Werner [21] in connection with conformal invariance and the Schramm-Loewner evolution (SLE), and named Brownian loop soup. A discrete version was introduced by Lawler and Trujillo Ferreras [19] who call it the random walk loop soup, and was studied extensively by Le Jan $[22,25]$ who showed a connection between the vertex-occupation field of the loop soup and the square of the discrete Gaussian free field. For a particular value of the intensity of the Poisson process, the random walk loop soup is exactly the lattice loop model mentioned above whose partition function coincides with that of the discrete Gaussian free field (up to a multiplicative constant).

For reasons discussed later in this introduction, in this paper, we introduce a new ingredient to the loop soup recipe: a non-backtracking condition on the loops. We show that the non-backtracking loop soup possesses a spatial Markov property, discussed in Sects. 2 and 3, which implies that the loop soup induces a Markovian edge-occupation field, which in turn implies that the field has a Gibbs distribution. Quite remarkably, the Hamiltonian can be written explicitly as a sum of local terms, and the partition function and the free energy density of the occupation field can be computed exactly in any dimension. These computations rely on the fact that, for this as for any loop soup, the partition function can be written in terms of a determinant. In the translation invariant case, the relevant determinant can be calculated and the free energy density of the field can be written in closed form on the $d$-dimensional torus for any $d$, as mentioned at the beginning of this introduction. 


\subsection{Motivations and Relations to Other Results}

Adding a non-backtracking condition to the definition of the loop soup reduces the number of walks and changes the partition function of the model. It turns out that the partition function of the non-backtracking loop soup computes the Ihara (edge) zeta function $[14,16,30,33]$ of the graph where the loop soup is defined, and this is one of the reasons the non-backtracking loop soup may be of interest. One can also still find a connection with the discrete Gaussian free field, as in the backtracking case, but this time in a less direct way via the Ihara zeta function, as explained in Sect. 4.

Another reason for studying non-backtracking loop soups is that they appear naturally in the Kac-Ward representation [17] of the Ising model in two dimensions, which can be expressed in terms of a Poissonian soup of nonbacktracking loops similar to the one studied in this paper but with signed weights (see [18]).

It is also worth mentioning the intriguing connection with spin networks, a concept introduced by Roger Penrose [28] which has applications to quantum gravity and appears also in conformal and topological quantum field theory (see, e.g., $[10,11,29]$ ). The non-backtracking loop soup studied in this paper generates spin network configurations with a certain Gibbs distribution, so it can be regarded as a statistical mechanical model on spin networks.

In two dimensions, the non-backtracking loop soup should be related to the Brownian loop soup of Lawler and Werner, and to SLE. Some evidence for this is contained in [12] where it is shown that, although with a different diffusion constant, the non-backtracking random walk satisfies a functional central limit theorem like the simple random walk.

A posteriori, a further compelling reason, already mentioned at the beginning of the introduction, for studying the non-backtracking loop soup is that its edge-occupation field provides a new Gibbsian model that is solvable in any dimension. In view of the role that exactly solvable models have played in one and two dimensions, the model we introduce in this paper may offer some insight into critical behavior in dimensions higher than two, where conformal invariance has so far not played the same role as in two dimensions.

Another interesting aspect of the non-backtracking loop soup studied in this paper is its spatial Markov property (see Theorem 3.3). This property is particularly interesting because it is not generic in the sense that multiplying the loop measure in the definition of the loop soup by any constant results in the loss of the Markov property. We point out, though, that the non-backtracking condition is not necessary to have the Markov property, as shown by Werner in reference [34], posted shortly after the first version of this paper appeared. Thus, the edge-occupation field of the corresponding backtracking loop soup is also Gibbsian. ${ }^{1}$ In that case, the partition function of the model is of course already known since it coincides with that of the discrete

\footnotetext{
${ }^{1}$ In the backtracking case, the Gibbsiannes of the occupation field can be established without reference to the Markov property of the loop soup, using instead its connection to the Gaussian free field, as done by Le Jan in $[23,25]$.
} 
Gaussian free field. (On the torus, the latter was calculated, for example, by Berlin and Kac [5].) We note that the spatial Markov property obtained by Werner in [34] in the case of backtracking loops can be proved also using the methods of this paper, as discussed after the proof of Theorem 3.3.

\section{Discussion of the Main Results}

We introduce and study a Poissonian model of non-backtracking loops and arcs on graphs in dimension $d \geq 2$, and the associated edge-occupation field, given by the number of visits to each edge by the collection of loops and arcs. Adopting the language of $[20,21]$, we refer to this model as a loop soup, even though the "soup" contains in general both loops and arcs that start and end at boundary edges. For simplicity, in this section, we focus only on loops; precise and more general definitions are given in the next section.

Consider a connected simple graph $\mathcal{G}=(V, E)$ and associate a positive weight $x_{e}$ to each edge $e \in E$. A (non-backtracking) loop $\ell$ is a closed walk on the edges of $\mathcal{G}$ considered up to cyclic shifts and reversal. A loop is said to have multiplicity $m_{\ell}$ if it can be obtained as the concatenation of $m_{\ell}$ copies of the same loop, and $m_{\ell}$ is the largest such number. A loop $\ell$ with multiplicity $m_{\ell}$ is assigned weight $\mu(\ell)=\frac{1}{m_{\ell}} \prod x_{e}$, where the product is taken over all edges traversed by $\ell$.The loop soup $\mathcal{L}$ studied in this paper is a Poisson point process on the space of non-backtracking loops with intensity measure $\mu$. The edge-occupation field $N_{\mathcal{L}}=\left(N_{\mathcal{L}}(e)\right)_{e \in E}$ induced by the loop soup is given by the total number of visits of the loops to each edge of $\mathcal{G}$.

Our main results concern both the loop soup itself and the induced edgeoccupation field.

1. The loop soup has a spatial Markov property such that, conditionally on the value of the edge-occupation field on a boundary set that splits the graph into two parts, the distributions of the loops and arcs contained in the two parts are independent of each other (Theorem 3.3).

2. The edge-occupation field has a Gibbs distribution (Theorem 3.1) whose Hamiltonian can be written explicitly (Eq. (3.1)) and whose partition function can be expressed as

$$
Z=\sum_{N} \prod_{v \in V} C_{v} \prod_{e \in E} \frac{x_{e}^{N(e)}}{N(e) !},
$$

where the sum runs over all spin network [28] configurations $N$ and $C_{v}=$ $C_{v}(N)$ is a suitable function of $(N(e))_{e \ni v}$.

3. The partition function can be expressed as a determinant and related to the Ihara zeta function and to the partition function of a discrete Gaussian free field (Sect. 4).

(4) In the homogeneous case, $x_{e} \equiv x$, on a finite $\delta$-regular graph, $Z<\infty$ if $x<1 /(\delta-1)$ and $Z$ diverges for $x=1 /(\delta-1)$ (Corollary 4.4).

In the case of translation invariant weights $x_{e}$, we have the following exact results. 
(5) The partition function of the occupation field can be computed explicitly on the $d$-dimensional torus in any dimension $d \geq 2$ (Corollary 5.2).

(6) The free energy density $f$ of the occupation field on $\mathbb{Z}^{d}$ can be computed explicitly for any dimension $d \geq 2$ as the thermodynamic limit of the free energy density on the $d$-dimensional torus (Corollary 5.3).

In the homogeneous case, $x_{e}=x \forall e \in E$, we have the following exact results on $\mathbb{Z}^{d}$.

(7) The $d$-dimensional free energy density $f(x)$ is finite for $x<1 /(2 d-1)$, and has a singularity as $x \nearrow 1 /(2 d-1)$ which depends on the dimension $d$ (Corollary 5.4).

(8) The truncated two-point function decays exponentially for $x<1 /(2 d-1)$ (Corollary 7.3).

Points (7) and (8) show that the edge-occupation field undergoes a sharp phase transition and that the critical point can be explicitly computed and the critical behavior analyzed for periodic graphs and lattices. This is done by studying the free energy density in the thermodynamic limit and by deriving expressions for the truncated two-point function.

In addition to the results mentioned above, Sects. 6 and 7 contain more results on the distribution and on the two-point function of the occupation field, respectively.

In the last section of the paper, we use the loop soup to define a spin model on the vertices of the dual of a planar graph $\mathcal{G}$. The spin model can be shown to be reflection positive using the Markov nature of the edge-occupation field. In two dimensions, we conjecture that the scaling limit of the spin field is one of the conformal fields introduced in [9] (see also [32]). We note that the analogous spin model generated by an ordinary loop soup was introduced by Le Jan [25] and is also reflection positive.

\section{The non-backtracking loop soup}

Let $\mathcal{G}=(V, E)$ be a connected graph, and let $\vec{E}$ be the set of its directed edges. For a directed edge $\vec{e}=\left(t_{\vec{e}}, h_{\vec{e}}\right) \in \vec{E},-\vec{e}=\left(h_{\vec{e}}, t_{\vec{e}}\right) \in \vec{E}$ is its reversal, and $e=\left\{t_{\vec{e}}, h_{\vec{e}}\right\} \in E$ its undirected version. We assume that the graph is equipped with a positive edge weight $x_{e}$ for each $e \in E$. By $\partial \mathcal{G}$ we denote the boundary of $\mathcal{G}$, i.e., the (possibly empty) set of edges incident on a vertex of degree one.

A (non-backtracking) walk $\vec{\omega}$ of length $|\vec{\omega}|=n \geq 1$ is a sequence of directed edges $\vec{\omega}=\left(\vec{\omega}_{1}, \ldots, \vec{\omega}_{n+1}\right)$ such that $t_{\vec{\omega}_{i+1}}=h_{\vec{\omega}_{i}}$ and $\vec{\omega}_{i+1} \neq-\vec{\omega}_{i}$ for $1 \leq i \leq n$. Note that the length of a walk is the number of steps the walk makes between the edges rather than the number of edges itself. By $\vec{\omega}^{-1}=\left(-\vec{\omega}_{|\vec{\omega}|+1}, \ldots,-\vec{\omega}_{1}\right)$ we denote its reversal, and for two walks $\vec{\omega}, \vec{\omega}^{\prime}$ such that $\vec{\omega}|\vec{\omega}|+1=\vec{\omega}_{1}^{\prime}$, we define the concatenation

$$
\vec{\omega} \oplus \vec{\omega}^{\prime}=\left(\vec{\omega}_{1}, \ldots, \vec{\omega}_{|\vec{\omega}|+1}, \vec{\omega}_{2}^{\prime}, \ldots, \vec{\omega}_{\left|\vec{\omega}^{\prime}\right|+1}^{\prime}\right) .
$$

Rooted loops are walks starting and ending at the same directed edge, i.e., $\vec{\omega}_{1}=\vec{\omega}_{|\vec{\omega}|+1}$. The multiplicity of a rooted loop $\vec{\omega}$, denoted by $m_{\vec{\omega}}$, is the 
largest number $m$ such that $\vec{\omega}$ is the $m$-fold concatenation of some rooted loop $\vec{\omega}^{\prime}$ with itself. An unoriented walk is a walk without a specified direction of traversal, i.e. a two-element equivalence class under the relation $\vec{\omega} \sim \vec{\omega}^{-1}$. An arc is an unoriented walk starting and ending on $\partial G$. Arcs will be denoted by $\alpha$. Unrooted loops are equivalence classes of loops under the cyclic shift relation $\vec{\omega} \sim\left(\vec{\omega}_{i}, \vec{\omega}_{i+1}, \ldots, \vec{\omega}_{|\vec{\omega}|}, \vec{\omega}_{1}, \vec{\omega}_{2}, \ldots, \vec{\omega}_{i}\right)$. Unrooted unoriented loops will be simply referred to as loops and will be denoted by $\ell$.

With a slight abuse of notation, if a function $f$ defined on walks is invariant under reversal, then $f(\alpha)$ is the evaluation of $f$ at any of the two representatives of the arc $\alpha$. Similarly, if a function $g$ defined on rooted loops is invariant under reversal and cyclic shift, $g(\ell)$ is the evaluation of $g$ at any representative of $\ell$. The weight of a walk $\vec{\omega}$ is

$$
x(\vec{\omega})=\prod_{i=1}^{|\vec{\omega}|} \sqrt{x_{\omega_{i}} x_{\omega_{i+1}}} .
$$

The loop and arc measures are given by

$$
\mu(\ell)=\frac{x(\ell)}{m_{\ell}} \quad \text { and } \quad \mu_{\partial}(\alpha)=x(\alpha) .
$$

We note that $1 / m_{\ell}=\rho_{\ell} /|\ell|$, where $\rho_{\ell}$ is the number of rooted oriented loops in the equivalence class $\ell$. This fact is used in Sect. 4 in the proof of Lemma $4.1 .^{2}$

By $\mathcal{L}$ we will denote a realization of a Poisson point process with intensity measure $\mu$, and by $\mathcal{A}$ a realization of a Poisson point process with intensity measure $\mu_{\partial}$. We will write $\mathcal{S}=\mathcal{L} \cup \mathcal{A}$, where $\mathcal{L}$ and $\mathcal{A}$ are independent. The partition function of $\mathcal{L}$ is

$$
Z_{\mathcal{L}}=\sum_{L} w(L)=\sum_{L} \prod_{\ell \in L} \frac{\mu(\ell)^{\# \ell}}{(\# \ell) !},
$$

where the sum is taken over all multi-sets $L$ of loops, called loop configurations, and where $\# \ell$ is the number of occurrences of $\ell$ in $L$. The second equality defines a weight function $w$ on the space of loop configurations. Similarly, the partition function of $\mathcal{A}$ is

$$
Z_{\mathcal{A}}=\sum_{A} w(A)=\sum_{A} \prod_{\alpha \in A} \frac{\mu_{\partial}(\alpha)^{\# \alpha}}{(\# \alpha) !}
$$

where the sum is taken over all multi-sets of $\operatorname{arcs} A$, called arc configurations, and where $w$ is the weight function on arc configurations. The partition function of $\mathcal{S}$ is given by $Z_{\mathcal{S}}=Z_{\mathcal{L}} Z_{\mathcal{A}}$. We will only consider the cases where $Z_{\mathcal{L}} Z_{\mathcal{A}}<\infty$. A multi-set of loops and arcs will be called a soup configuration or simply a configuration. In particular, loop and arc configurations are soup configurations. The weight $w(S)$ of a soup configuration $S$ is the product $w(L) w(A)$ of the weights of its loop configuration $L$ and its arc configuration $A$. With a slight abuse of notation we use the same symbol $w$ for the weight functions of loop configurations, arc configurations and soup configurations.

2 The factor $1 /|\ell|$ was overlooked in Lemma 1.2 of [7], but the mistake was later corrected in [8]. 
A spin network in the sense of Penrose [28] is an assignment of a natural number $N(e)$ to each edge $e$ in such a way that if $e_{1}, \ldots, e_{k}$ are the edges incident on vertex $v$, then the sum $\sum_{i=1}^{k} N_{S}\left(e_{i}\right)$ is even and is not smaller than $2 \max _{i=1, \ldots, k} N_{S}\left(e_{i}\right)$. For a soup configuration $S$, we define $N_{S}=\left(N_{S}(e)\right)_{e \in E}$ to be the network (or edge-occupation field) induced by $S$, i.e., the total number of visits of the walks from $S$ to each edge of $\mathcal{G}$. One can check that, since the walks are non-backtracking, the induced network is a spin network.

One of the main results of this paper is that the distribution of the random network $N_{\mathcal{S}}$ with prescribed boundary conditions $\xi=(N(e))_{e \in \partial \mathcal{G}}$ (in particular, the distribution of $N_{\mathcal{L}}$ for zero boundary conditions) is given by a Gibbs distribution with a local Hamiltonian.

More precisely, suppose that $e_{1}, \ldots, e_{k}$ are all the edges incident on vertex $v$ and imagine replacing edge $e_{i}$ by $N_{S}\left(e_{i}\right)$ distinct, colored edges. Assume that each colored edge incident on $v$ has a unique color and let $C_{v}$ be the number of different ways in which those edges can be connected in such a way that each colored edge corresponding to $e_{i}$ is connected to a colored edge corresponding to $e_{j}$ for some $j \neq i$. (It is clear that one can always connect all colored edges in this way since, after all, $N_{S}\left(e_{i}\right)$ is the number of visits to edge $e_{i}$ of the non-backtracking loops and arcs from the soup.) If $\mathcal{G}=(V, E)$ is finite, we can define the Hamiltonian $\mathcal{H}=\mathcal{H}\left(N_{S}\right)=\sum_{v \in V} H_{v}$ where, for each vertex $v$ with edges $e_{1}, \ldots, e_{k}$ incident on it,

$$
H_{v}=-\log \left(\frac{C_{v}}{\sqrt{\prod_{i=1}^{k} N_{S}\left(e_{i}\right) !}} \prod_{i=1}^{k} x_{e_{i}}^{N_{S}\left(e_{i}\right) / 2}\right) .
$$

Theorem 3.1 (Gibbs distribution). If the graph $\mathcal{G}$ is finite and $\mathcal{S}$ is a soup of loops and arcs in $\mathcal{G}$ such that $Z_{\mathcal{S}}<\infty$, then the distribution of the edgeoccupation field induced by $\mathcal{S}$ is a Gibbs distribution with Hamiltonian $\mathcal{H}$; that is, the edge-occupation configuration $N_{S}$ has probability

$$
\frac{1}{Z} e^{-\mathcal{H}\left(N_{S}\right)},
$$

where

$$
Z=\sum_{N} e^{-\mathcal{H}(N)}=\sum_{N} \prod_{v \in V} C_{v} \prod_{e \in E} \frac{x_{e}^{N(e)}}{N(e) !}
$$

with the sums running over all network configurations $N$.

We note that the partition function (3.2) is reminiscent of the random current representation used by Aizenman [1,2] (see also [3] for a more recent application).

If $\mathcal{G}$ is a trivalent graph, the combinatorics is simple and one can compute $C_{v}$. (Penrose uses trivalent graphs to define spin networks precisely for this reason, although the concept is more general-see [28].) Let $e_{1}, e_{2}, e_{3}$ be the edges incident on some vertex $v$ of $\mathcal{G}$, with occupation values $N\left(e_{1}\right), N\left(e_{2}\right)$ and $N\left(e_{3}\right)$, and define $N_{12}=\frac{N\left(e_{1}\right)+N\left(e_{2}\right)-N\left(e_{3}\right)}{2}, N_{13}=\frac{N\left(e_{1}\right)+N\left(e_{3}\right)-N\left(e_{2}\right)}{2}$, $N_{23}=\frac{N\left(e_{2}\right)+N\left(e_{3}\right)-N\left(e_{1}\right)}{2}$. (These are the unique solutions of the system of 
linear equations $N_{12}+N_{13}=N\left(e_{1}\right), N_{12}+N_{23}=N\left(e_{2}\right), N_{13}+N_{23}=N\left(e_{3}\right)$.) It is easy to see that $C_{v}=\frac{N\left(e_{1}\right) ! N\left(e_{2}\right) ! N\left(e_{3}\right) !}{N_{12} ! N_{13} ! N_{23} !}$ and consequently

$$
H_{v}=-\log \left(\frac{\sqrt{N\left(e_{1}\right) ! N\left(e_{2}\right) ! N\left(e_{3}\right) !}}{N_{12} ! N_{13} ! N_{23} !} x_{e_{1}}^{N\left(e_{1}\right) / 2} x_{e_{2}}^{N\left(e_{2}\right) / 2} x_{e_{3}}^{N\left(e_{3}\right) / 2}\right) .
$$

Theorem 3.1 is a consequence of the conditional factorization property of the law of $\mathcal{S}$, which implies that the soup possesses a spatial Markov property. In other words, the edge-occupation field is a Markov field. A natural way to express this property is to cut loops into arcs, and we will now make precise what we mean by that. Suppose that we fix a set of edges $H \subset E$. By $\mathcal{G}_{H}$ we denote the modified graph $\mathcal{G}$ where the edges from $H$ are cut into half, i.e., for each $e=\{u, v\} \in H$, we remove $e$ from the edge-set, and add two new edges, $\left\{u, u^{\prime}\right\}$ and $\left\{v^{\prime}, v\right\}$, called half-edges and incident on two new vertices $u^{\prime}, v^{\prime} \notin V$ with $u^{\prime} \neq v^{\prime}$. The weight of each half-edge is equal to the weight of the removed edge it replaces. Note that the half-edges belong to $\partial \mathcal{G}_{H}$. Each loop or arc in $\mathcal{G}$ visiting $H$, when cut along $H$, gives rise to a multi-set of arcs in $\mathcal{G}_{H}$, corresponding to its maximal subwalks which visit only edges from $E \backslash H$, except for their first and last edge. These arcs are the excursions the walks make outside of $H$. If a walk does not visit $H$, then it is not affected when the edges of $H$ are cut in two. Given a configuration $S$ in $\mathcal{G}$, we define $S_{\mathcal{G}_{H}}$ to be the configuration in $\mathcal{G}_{H}$ resulting from cutting the walks from $S$ (taken with multiplicities) along $H$.

Lemma 3.2 (Conditional factorization). For a set of edges $H$, and a configuration $S$,

$$
\sum_{T: T_{\mathcal{G}_{H}}=S_{\mathcal{G}_{H}}} w(T)=w\left(S_{\mathcal{G}_{H}}\right) \prod_{e \in H} N_{S}(e) !
$$

Proof. Note that all $T$ with $T_{\mathcal{G}_{H}}=S_{\mathcal{G}_{H}}$ differ only in the way the arcs of $T_{\mathcal{G}_{H}}$ are connected to each other along the edges in $H$. It is hence enough to consider the case when all walks in $S$ visit $H$ and, therefore, $S_{\mathcal{G}_{H}}$ consists only of arcs. This is because the contribution of the loops from $T \cap T_{\mathcal{G}_{H}}$ to $w(T)$ is the same for all $T$ with $T_{\mathcal{G}_{H}}=S_{\mathcal{G}_{H}}$ and is equal to the total loop contribution to $w\left(S_{\mathcal{G}_{H}}\right)$.

We will prove the result by showing that both the l.h.s. and the r.h.s. of the equality are proportional to the number of ways one can assign colors to the visits of loops and arcs of a configuration to the edges of $H$. We will now make precise what we mean by this. To this end, let $\mathcal{G}_{H}^{\prime}$ be the graph obtained from $\mathcal{G}_{H}$ by connecting, for each $e \in H$, the degree-one vertices of the half-edges replacing $e$ with $N_{S}(e)$-distinguishable parallel edges (see Fig. 1). We can think of the new edges as being colored by $N_{S}(e)$ different colors. Consider configurations in $\mathcal{G}_{H}^{\prime}$ which visit each of the colored edges exactly once. Each such configuration maps to a configuration in $\mathcal{G}$ by forgetting the colors and identifying the colored edges for each $e \in H$. This mapping produces a configuration $T$ in $\mathcal{G}$ that satisfies $\left.N_{T}\right|_{H}=\left.N_{S}\right|_{H}$. The mapping is many-toone, and we think of the cardinality of the preimage of each configuration $T$ in 


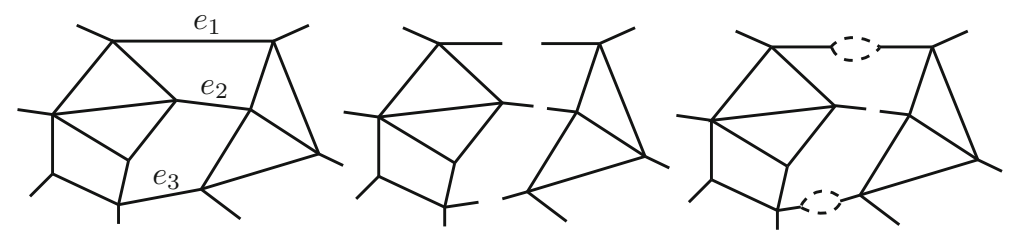

Figure 1. Graphs $\mathcal{G}$ with $H=\left\{e_{1}, e_{2}, e_{3}\right\}, \mathcal{G}_{H}$, and $\mathcal{G}_{H}^{\prime}$ for $S$ with $N_{S}\left(e_{1}\right)=2, N_{S}\left(e_{2}\right)=0, N_{S}\left(e_{1}\right)=2$

$\mathcal{G}$ as the number of ways one can assign colors to the visits of loops and arcs of $T$ to the edges of $H$. We will now show that this number is proportional to $w(T)$.

To this end, fix a configuration $T$ in $\mathcal{G}$ such that $T_{\mathcal{G}_{H}}=S_{\mathcal{G}_{H}}$. For each loop in $T$ choose a root and an orientation, and if a loop is repeated in $T$, choose the same root and the same orientation for every copy of the loop. Moreover, order the copies of each repeated loop or arc in $T$ so that each copy becomes distinguishable. Call $\tilde{T}$ the resulting collection of distinguishable arcs and distinguishable, oriented, rooted loops. Since all steps of the walks in $\tilde{T}$ are distinguishable, for each $e \in H$ we have $N_{S}(e)$ ! different ways of assigning colors to the visits of the walks in $\tilde{T}$ to $e$, by which we mean that there are $N_{S}(e)$ ! different ordered configurations of arcs and directed rooted loops in $\mathcal{G}_{H}^{\prime}$ which map to $\tilde{T}$ after collapsing the colored edges and forgetting the colors (but not the roots and orientations of loops). This gives a total of $\prod_{e \in H} N_{S}(e)$ !, which, however, is an overshoot due to the possible presence of identical copies of loops and arcs, and of loops with nontrivial periods. What is left to do is to account for the multiplicities coming from identifying the ordered copies of loops and arcs, and from identifying the periodic shifts of loops with nontrivial periods. Note that each loop in $\mathcal{G}$ has the same number of different directed versions (exactly two) as any of the corresponding loops in $\mathcal{G}_{H}^{\prime}$; hence, one can choose an orientated representative of each loop in $T$ in the counting of unoriented loops (see Remark 3.4). This gives

$$
\frac{\prod_{e \in H} N_{S}(e) !}{\prod_{\ell \in T}(\# \ell) ! m_{\ell}^{\# \ell} \prod_{\alpha \in T}(\# \alpha) !}=\frac{\prod_{e \in H} N_{S}(e) !}{\prod_{\ell \in T} x(\ell) \# \ell} \prod_{\alpha \in T} x(\alpha)^{\# \alpha} w(T)
$$

for the number of ways one can assign colors to the visits of loops and arcs of $T$ to the edges of $H$. Using (3.3), the total number of ways one can assign colors to the visits of loops and arcs to the edges of $H$, for all configurations $T$ satisfying $T_{\mathcal{G}_{H}}=S_{\mathcal{G}_{H}}$, can be written as

$$
\sum_{T: T_{\mathcal{G}_{H}}=S_{\mathcal{G}_{H}}} \frac{w(T)}{\prod_{\ell \in T} x(\ell)^{\# \ell} \prod_{\alpha \in T} x(\alpha)^{\# \alpha}} \prod_{e \in H} N_{S}(e) !
$$

Let us now derive a different expression for this number, this time by counting the number of ways one can connect the arcs from $S_{\mathcal{G}_{H}}$ into loops in 
$\mathcal{G}_{H}^{\prime}$ in such a way that each colored edge is used only once. To this end, take an edge $e=\{u, v\}$ from $H$ and consider the two directed half-edges, $\vec{e}_{1}=\left(v^{\prime}, v\right)$ and $\vec{e}_{2}=\left(u^{\prime}, u\right)$, and the $N_{S}(e)$ colored edges in $\mathcal{G}_{H}^{\prime}$ corresponding to $e$. For the purpose of this proof, we will consider directed arcs. Let $\vec{A}\left(\vec{e}_{i}\right)$ be the multi-set of all directed versions of the arcs from $S_{\mathcal{G}_{H}}$ starting at $\vec{e}_{i}, i=1,2$. One has

$$
\sum_{\vec{\alpha} \in \vec{A}\left(\vec{e}_{i}\right)} \# \vec{\alpha}=N_{S}(e) \quad \text { for } \quad i=1,2,
$$

where $\# \vec{\alpha}$ is the multiplicity of the directed arc $\vec{\alpha}$ in $\vec{A}\left(\vec{e}_{i}\right)$ (which is equal to the multiplicity of its undirected version $\alpha$ in $S_{\mathcal{G}_{H}}$ ). We now distribute the $N_{S}(e)$ colors between the directed arcs in $\vec{A}\left(\vec{e}_{i}\right), i=1,2$. Since the arcs have multiplicities $\# \vec{\alpha}$, there are exactly

$$
\frac{N_{S}(e) !}{\prod_{\vec{\alpha}_{1} \in \vec{A}\left(\vec{e}_{1}\right)}\left(\# \vec{\alpha}_{1}\right) !} \times \frac{N_{S}(e) !}{\prod_{\vec{\alpha}_{2} \in \vec{A}\left(\vec{e}_{2}\right)}\left(\# \vec{\alpha}_{2}\right) !}
$$

such assignments. If we take the product over $H$ and use the fact that for each arc $\vec{\alpha}$ its reversal $\vec{\alpha}^{-1}$ also appears in the product, we arrive at

$$
\frac{\prod_{e \in H} N_{S}(e) !^{2}}{\prod_{\alpha \in S_{\mathcal{G}_{H}}}(\# \alpha) !^{2}}
$$

where $\# \alpha$ is the multiplicity of $\alpha$ in $S_{\mathcal{G}_{H}}$. This is the number of all possible assignments of colors to the directed arcs. We now want to forget the orientation of the arcs, so, for each arc $\alpha$, we need to pair up the opposite directed, colored arcs $\vec{\alpha}$ and $\vec{\alpha}^{-1}$. Since we can pair any colored arc $\vec{\alpha}$ with any colored $\operatorname{arc} \vec{\alpha}^{-1}$, we have $(\# \alpha)$ ! different pairings. Hence, we obtain

$$
\frac{\prod_{e \in H} N_{S}(e) !^{2}}{\prod_{\alpha \in S_{\mathcal{G}_{H}}}(\# \alpha) !}=\frac{w\left(S_{\mathcal{G}_{H}}\right)}{\prod_{\alpha \in S_{\mathcal{G}_{H}}} x(\alpha) \# \alpha} \prod_{e \in H} N_{S}(e) !^{2}
$$

for the number of all possible ways of connecting the arcs from $S_{H}$ in such a way that each colored edge is used once. This provides a second expression for (3.4).

We remind the reader that we need only consider the case when all walks in $S$ visit $H$ and hence $S_{\mathcal{G}_{H}}$ consists only of arcs. We also note that, for every $T$ such that $T_{\mathcal{G}_{H}}=S_{\mathcal{G}_{H}}$, we have the identity

$$
\prod_{\ell \in T} x(\ell)^{\# \ell} \prod_{\alpha \in T} x(\alpha)^{\# \alpha}=\prod_{\alpha \in S_{\mathcal{G}_{H}}} x(\alpha)^{\# \alpha} .
$$

Using this identity and comparing (3.4) with (3.5) concludes the proof.

Given a subgraph $\mathcal{G}_{1}$ of $\mathcal{G}_{H}$ which is a union of a number of connected components of $\mathcal{G}_{H}$, we define $S_{\mathcal{G}_{1}}$ to be $S_{\mathcal{G}_{H}}$ restricted to walks in $\mathcal{G}_{1}$. If $\xi$ : $\partial \mathcal{G} \rightarrow \mathbb{N}_{\geq 0}$ are boundary conditions, then we write $\mathbb{P}_{\mathcal{G}, \xi}$ for the probability measure governing $\mathcal{S}$ defined on $G$ and conditioned to satisfy $\left.N_{\mathcal{S}}\right|_{\partial \mathcal{G}}=\xi$. 
Theorem 3.3 (Spatial Markov property). Let $H$ be a set of edges of $\mathcal{G}$, and let $\mathcal{G}_{1}$ be one of the connected components of $\mathcal{G}_{H}$. Let $S$ be a configuration in $\mathcal{G}$. Then, for all boundary conditions $\xi=(N(e))_{e \in \partial \mathcal{G}}$ on $\partial \mathcal{G}$,

$$
\begin{aligned}
\mathbb{P}_{\mathcal{G}, \xi}\left(\mathcal{S}_{\mathcal{G}_{1}}=S_{\mathcal{G}_{1}} \mid \mathcal{S}_{\mathcal{G}_{H} \backslash \mathcal{G}_{1}}=S_{\mathcal{G}_{H} \backslash \mathcal{G}_{1}}\right) & =\mathbb{P}_{\mathcal{G}_{, \xi}}\left(\mathcal{S}_{\mathcal{G}_{1}}=S_{\mathcal{G}_{1}}\left|N_{\mathcal{S}}\right|_{\partial \mathcal{G}_{1}}=\left.N_{S}\right|_{\partial \mathcal{G}_{1}}\right) \\
& =\mathbb{P}_{\mathcal{G}_{1}, \xi_{1}}\left(\mathcal{S}=S_{\mathcal{G}_{1}}\right),
\end{aligned}
$$

where $\xi_{1}$ are boundary conditions on $\partial \mathcal{G}_{1}$ given by

$$
\xi_{1}=\left\{\begin{array}{lll}
\xi & \text { on } & \partial \mathcal{G}_{1} \cap \partial \mathcal{G}, \\
\left.N_{S}\right|_{\partial \mathcal{G}_{1}} & \text { on } & \partial \mathcal{G}_{1} \backslash \partial \mathcal{G} .
\end{array}\right.
$$

Proof. From Lemma 3.2 and the factorization property of the Poisson point process weights, it follows that

$$
\begin{aligned}
\mathbb{P}_{\mathcal{G}, \xi} & \left(\mathcal{S}_{\mathcal{G}_{1}}=S_{\mathcal{G}_{1}} \mid \mathcal{S}_{\mathcal{G}_{H} \backslash \mathcal{G}_{1}}=S_{\mathcal{G}_{H} \backslash \mathcal{G}_{1}}\right) \\
= & \mathbb{P}_{\mathcal{G}_{, \xi}}\left(\mathcal{S}_{\mathcal{G}_{H}}=S_{\mathcal{G}_{H}}\right) / \mathbb{P}_{\mathcal{G}_{, \xi}}\left(\mathcal{S}_{\mathcal{G}_{H} \backslash \mathcal{G}_{1}}=S_{\mathcal{G}_{H} \backslash \mathcal{G}_{1}}\right) \\
= & \sum_{T: T_{\mathcal{G}_{H}}=S_{\mathcal{G}_{H}}} w(T) / \sum_{T: T_{\mathcal{G}_{H} \backslash \mathcal{G}_{1}}=S_{\mathcal{G}_{H} \backslash \mathcal{G}_{1}}} w(T) \\
= & w\left(S_{\mathcal{G}_{1}} \cup S_{\mathcal{G}_{H} \backslash \mathcal{G}_{1}}\right) / \sum_{T_{1}: N_{T_{1}}\left|\partial \mathcal{G}_{1}=N_{S}\right|_{\partial \mathcal{G}_{1}}} w\left(T_{1} \cup S_{\mathcal{G}_{H} \backslash \mathcal{G}_{1}}\right) \\
= & w\left(S_{\mathcal{G}_{1}}\right) / \sum_{T_{1}: N_{T_{1}}\left|\partial \mathcal{G}_{1}=N_{S}\right|_{\partial \mathcal{G}_{1}}} w\left(T_{1}\right) \\
= & \mathbb{P}_{\mathcal{G}_{1}, \xi_{1}}\left(\mathcal{S}=S_{\mathcal{G}_{1}}\right),
\end{aligned}
$$

where $T_{1}$ denotes a configuration in $\mathcal{G}_{1}$.

Remark 3.4. The spatial Markov property in the theorem above holds also for the following soup of loops and arcs where backtracking is allowed. Consider first a soup of oriented loops and arcs (possibly backtracking) with loop measure $\frac{1}{2} \mu$ and arc measure $\frac{1}{2} \mu_{\partial}$, and then forget the orientations of the loops and arcs. For loops and arcs with two oriented versions, including in particular all non-backtracking loops and arcs, this is equivalent to considering unoriented loops and arcs from the start but with measures $\mu$ and $\mu_{\partial}$, respectively. But for loops and arcs that have a single oriented version (and are, therefore, necessarily backtracking), the factor $1 / 2$ in the measures remains even after forgetting the orientation. Keeping this in mind, one can repeat the arguments and redo the calculations in the proof of Theorem 3.3, and obtain the same spatial Markov property for this new soup. This is a slight generalization of Proposition 4 in [34] where a similar soup is considered but containing only loops.

We conclude this section with a proof of Theorem 3.1. We first need to state a combinatorial lemma which follows from the arguments contained in the proof of Lemma 3.2. For a network $N$ and a soup configuration $S$, we write $S \sim N$ if $N_{S}=N$. Let $\mathcal{G}_{N}^{\prime}$ be the extended graph $\mathcal{G}$ where each edge $e \in E$ is replaced by $N(e)$ parallel edges. Consider configurations $S^{\prime}$ in $\mathcal{G}_{N}^{\prime}$ which 
visit each of these edges exactly once and do not visit any other edge. We write $\mathcal{S}^{\prime}(N)$ for the set of such configurations and $\left|\mathcal{S}^{\prime}(N)\right|$ for its cardinality. Note that for each $S^{\prime} \in \mathcal{S}^{\prime}(N)$, the configuration $S$ in $\mathcal{G}$ obtained from $S^{\prime}$ by identifying the parallel edges satisfies $S \sim N$.

Lemma 3.5. We have

$$
\left|\mathcal{S}^{\prime}(N)\right|=\prod_{e \in E} N(e) ! \sum_{S \sim N} \prod_{\ell \in S} \frac{1}{(\# \ell) ! m_{\ell}^{\# \ell}} \prod_{\alpha \in S} \frac{1}{(\# \alpha) !} .
$$

Proof. To obtain the identity it is enough to sum the l.h.s. of (3.3) over all configurations $S \sim N$ where $H$ is chosen to be the set of edges $e$ with $N(e)>$ 0 .

Proof of Theorem 3.1. The partition function of the soup can be written as

$$
\begin{aligned}
Z_{\mathcal{S}} & =\left(\sum_{L} \prod_{\ell \in L} \frac{\mu(\ell)^{\# \ell}}{(\# \ell) !}\right)\left(\sum_{A} \prod_{\alpha \in A} \frac{\mu_{\partial}(\alpha)^{\# \alpha}}{(\# \alpha) !}\right) \\
& =\sum_{S} \prod_{\ell \in S} \frac{x(\ell)^{\# \ell}}{(\# \ell) ! m_{\ell}^{\# \ell}} \prod_{\alpha \in S} \frac{x(\alpha)^{\# \alpha}}{(\# \alpha) !} \\
& =\sum_{N} \prod_{e \in E} x_{e}^{N(e)} \sum_{S \sim N} \prod_{\ell \in S} \frac{1}{(\# \ell) ! m_{\ell}^{\# \ell}} \prod_{\alpha \in S} \frac{1}{(\# \alpha) !} \\
& =\sum_{N}\left|\mathcal{S}^{\prime}(N)\right| \prod_{e \in E} \frac{x_{e}^{N(e)}}{N(e) !} \\
& =\sum_{N} \prod_{v \in V} C_{v} \prod_{e \in E} \frac{x_{e}^{N(e)}}{N(e) !} \\
& =\sum_{N} \prod_{v \in V} e^{-H_{v}} \\
& =\sum_{N} e^{-\mathcal{H}(N)} \\
& =Z
\end{aligned}
$$

where we have used the obvious identity $\left|\mathcal{S}^{\prime}(N)\right|=\prod_{v \in V} C_{v}$ and Lemma 3.5. To conclude the proof, we write

$$
\begin{aligned}
\sum_{S \sim N} \mathbb{P}_{\mathcal{G}}(S) & =\frac{1}{Z_{\mathcal{S}}} \sum_{S \sim N} \prod_{\ell \in S} \frac{x(\ell)}{(\# \ell) ! m_{\ell}^{\# \ell}} \prod_{\alpha \in S} \frac{x(\alpha)}{(\# \alpha) !} \\
& =\frac{1}{Z}\left|\mathcal{S}^{\prime}(N)\right| \prod_{e \in E} \frac{x_{e}^{N(e)}}{N(e) !} \\
& =\frac{1}{Z} \prod_{v \in V} C_{v} \prod_{e \in E} \frac{x_{e}^{N(e)}}{N(e) !}
\end{aligned}
$$




$$
\begin{aligned}
& =\frac{1}{Z} \prod_{v \in V} e^{-H_{v}} \\
& =\frac{1}{Z} e^{-\mathcal{H}(N)} .
\end{aligned}
$$

\section{Determinantal Formulas for the Partition Function}

In this section, we express the partition function of our model in terms of determinants of two different matrices, which, for certain values of the edge weights, involve transition matrices of some Markov processes. The process involved in the first determinantal formula is an asymmetric random walk on the directed edges of $\mathcal{G}$, and the one involved in the second formula is a random walk on the vertices of $\mathcal{G}$. As a consequence of the second determinantal formula, we derive a relation between the partition function of our model and that of the discrete Gaussian free field. Along the way, we also uncover a connection between the partition function of our model and the Ihara zeta function. In the next section, we will use the first determinantal formulas to do exact computations.

We assume that $\mathcal{G}$ is finite and connected and has no vertex of degree 1. For $\vec{e}, \vec{g} \in \vec{E}$, let

$$
\Lambda_{\vec{e}, \vec{g}}= \begin{cases}x_{e} & \text { if } h_{\vec{e}}=t_{\vec{g}} \text { and } t_{\vec{e}} \neq h_{\vec{g}} \\ 0 & \text { otherwise }\end{cases}
$$

and let $\rho(\Lambda)$ be the spectral radius of $\Lambda$. The next observation, a well-known result, is crucial and allows for an exact solution of our model.

Lemma 4.1. The partition function $Z_{\mathcal{L}}$ is finite if and only if $\rho(\Lambda)<1$, in which case

$$
Z_{\mathcal{L}}=\operatorname{det}^{-\frac{1}{2}}(\mathrm{Id}-\Lambda)
$$

where Id is the identity matrix indexed by $\vec{E}$.

Proof. Let $\theta_{i}, i=1,2, \ldots,|\vec{E}|$, be the eigenvalues of $\Lambda$, and let $\mathcal{W}_{\vec{e}, \vec{e}}^{n}$ be the set of all oriented walks of length $n$ starting and ending at the oriented edge $\vec{e}$. Using the definition of the loop measure, we have that for each $n$,

$$
\sum_{\ell:|\ell|=n} \mu(\ell)=\sum_{\ell:|\ell|=n} \frac{x(\ell)}{m_{\ell}}=\sum_{\vec{e} \in \vec{E}} \sum_{\vec{\omega} \in \mathcal{W}_{\vec{e}, \vec{e}}^{n}} \frac{x(\vec{\omega})}{2 n}=\frac{\operatorname{Tr} \Lambda^{n}}{2 n}=\sum_{i=1}^{|\vec{E}|} \frac{\theta_{i}^{n}}{2 n} .
$$

It follows that the first expression is summable over $n$ if and only if $\max _{i}\left|\theta_{i}\right|=$ $\rho(\Lambda)<1$. From the definition of the partition function, we have that

$$
Z_{\mathcal{L}}=\exp \left(\sum_{n=1}^{\infty} \sum_{\ell:|\ell|=n} \mu(\ell)\right)
$$


and hence the first part of the lemma follows. To finish the proof, we notice that

$-2 \ln Z_{\mathcal{L}}=-\sum_{n=1}^{\infty} \sum_{i=1}^{|\vec{E}|} \frac{\theta_{i}^{n}}{n}=\sum_{i=1}^{|\vec{E}|} \ln \left(1-\theta_{i}\right)=\ln \prod_{i=1}^{|\vec{E}|}\left(1-\theta_{i}\right)=\ln \operatorname{det}(\operatorname{Id}-\Lambda)$.

In the next section, we will use Lemma 4.1 to perform exact computations. First, however, we describe an interesting identity providing a different determinantal representation for the partition function. Such a determinantal representation was introduced in connection with the Ihara (edge) zeta function $[14,16,30]$, which is defined as the infinite product

$$
\zeta(\mathbf{x})=\prod_{\vec{\omega} \in \mathcal{P}}(1-x(\vec{\omega}))^{-1},
$$

where $\mathbf{x}=\left(x_{e}\right)_{e \in E}$ denotes the vector of edge weights and $\mathcal{P}$ is the collection of all oriented, unrooted, non-backtracking loops with multiplicity 1 . Note that in the general definition of the Ihara zeta function, the weights $x_{e}$ can be complex and can be different for the two opposite orientations of an undirected edge. By grouping together loops in (4.2) that are multiples of the same loop $\vec{\omega} \in \mathcal{P}$, we obtain that $Z_{\mathcal{L}}=\zeta^{\frac{1}{2}}(\mathbf{x})$, and hence by Lemma $4.1, \zeta^{-1}(\mathbf{x})=\operatorname{det}(\operatorname{Id}-\Lambda)$. (This is the content of Theorem 3 of [30] and Theorem 3.3 of [15].)

To state the alternative determinantal formula, we need to first define two matrices indexed by the vertices of $\mathcal{G}$. If $\|\mathbf{x}\|_{\infty}<1$, let $D$ be a diagonal matrix with entries

$$
D_{v, v}=\sum_{e \ni v} \frac{x_{e}^{2}}{1-x_{e}^{2}}
$$

and let

$$
A_{v, u}= \begin{cases}\frac{x_{e}}{1-x_{e}^{2}} & \text { if } e=\{u, v\} \in E \\ 0 & \text { otherwise }\end{cases}
$$

be a weighted adjacency matrix.

Lemma 4.2. For $\|\mathrm{x}\|_{\infty}$ small enough,

$$
Z_{\mathcal{L}}=\operatorname{det}^{-1 / 2}(\mathrm{Id}+D-A) \prod_{e \in E}\left(1-x_{e}^{2}\right)^{-1 / 2},
$$

where Id is the identity matrix indexed by $V$.

Proof. This result follows from Lemma 4.1 and Theorem 2 of [33], which generalizes previous results - see, e.g., [30] — to the case of non-equal edge weights considered in this lemma.

We will use Lemma 4.2 to relate the partition function of our model to that of a discrete Gaussian free field. To this end, attach a weight $c_{e} \geq 0$ to each edge $e \in E$ and a killing rate $k_{v} \geq 0$ to each vertex $v \in V$, and let $\lambda_{v}=\sum_{e \ni v} c_{e}+k_{v}$. The weights and killing rates induce a sub-Markovian transition matrix $P$ on the vertices of $\mathcal{G}$ with transition probabilities $p_{v, u}=\frac{c_{e}}{\lambda_{v}}$ if $e=\{v, u\} \in E$, and 0 otherwise. The transition matrix is $\lambda$-symmetric, 
meaning that $\lambda_{v} p_{v, u}=\lambda_{u} p_{u, v}$. One can introduce a "cemetery" state $\Delta$ and extend $P$ to a Markovian transition matrix $\bar{P}$ on $V \cup \Delta$ by setting $p_{v, \Delta}=\frac{k_{v}}{\lambda_{v}}$ and $p_{\Delta, \Delta}=1$. We call $\bar{P}$ the Markovian extension of $P$. We assume that the Green's function $G_{\bar{P}}(v, u)$ of the Markov chain associated to $\bar{P}$ (i.e., the expected number of visits to $u$ of the chain started at $v$ ) is finite. This condition is equivalent to saying that $\rho(P)<1$ or that the Markov chain is always absorbed in $\Delta$.

The discrete Gaussian free field on $\mathcal{G}$ associated with the transition matrix $\bar{P}$ is a collection $\left(\phi_{v}\right)_{v \in V}$ of mean-zero Gaussian random variables with covariance $\mathbb{E}\left(\phi_{v} \phi_{u}\right)=G_{\bar{P}}(v, u)$. It has a Gibbs distribution $e^{-H_{\bar{P}}^{G F F}} / Z_{\bar{P}}^{G F F}$ with Hamiltonian

$$
H_{\bar{P}}^{G F F}(\varphi)=\frac{1}{2} \sum_{\{v, u\} \in E} c_{\{v, u\}}\left(\varphi_{v}-\varphi_{u}\right)^{2}+\frac{1}{2} \sum_{v \in V} k_{v} \varphi_{v}^{2},
$$

from which it follows that its partition function, $Z_{\bar{P}}^{G F F}$, is given by a Gaussian integral and can, therefore, be represented in terms of a determinant, as follows:

$$
Z_{\bar{P}}^{G F F}=\prod_{v \in V}\left(\frac{2 \pi}{\lambda_{v}}\right)^{1 / 2} \operatorname{det}^{-\frac{1}{2}}(\mathrm{Id}-P)
$$

where Id is the identity matrix indexed by $V$.

Theorem 4.3. Let $\mathbf{x}$ be a vector of edge weights such that $\|\mathbf{x}\|_{\infty}<1$ and

$$
\sum_{e \ni v} \frac{x_{e}}{1+x_{e}} \leq 1 \quad \forall v \in V
$$

For $v \in V$, let

$$
\lambda_{v}^{*}=1+\sum_{e \ni v} \frac{x_{e}^{2}}{1-x_{e}^{2}}
$$

Then, the matrix

$$
P_{v, u}^{*}= \begin{cases}\frac{1}{\lambda_{v}^{*} \frac{x_{e}}{1-x_{e}^{2}}} & \text { if } e=\{v, u\} \in E \\ 0 & \text { otherwise }\end{cases}
$$

is a $\lambda^{*}$-symmetric, sub-Markovian transition matrix on $V$. Moreover, if $Z_{\mathcal{L}}<$ $\infty$

$$
Z_{\mathcal{L}}=(2 \pi)^{-|V| / 2} \prod_{e \in E}\left(1-x_{e}^{2}\right)^{-1 / 2} Z_{\bar{P}^{*}}^{G F F},
$$

where $\bar{P}^{*}$ is the Markovian extension of $P^{*}$.

Proof. The matrix $P^{*}$ is obviously $\lambda^{*}$-symmetric and an easy computation shows that the inequalities

$$
\sum_{e \ni v} \frac{x_{e}}{1+x_{e}} \leq 1 \quad \text { and } \quad \sum_{e \ni v} \frac{1}{\lambda_{v}^{*}} \frac{x_{e}}{1-x_{e}^{2}} \leq 1
$$


are equivalent. Hence, condition (4.4) guarantees that $P^{*}$ is sub-Markovian. Using Lemma 4.2, for $\|\mathbf{x}\|_{\infty}$ sufficiently small, we obtain

$$
\begin{aligned}
\left(Z_{\mathcal{L}}\right)^{-2} & =\prod_{e \in E}\left(1-x_{e}^{2}\right) \operatorname{det}(\operatorname{Id}+D-A) \\
& =\prod_{e \in E}\left(1-x_{e}^{2}\right) \prod_{v \in V}\left(1+\sum_{e \ni v} \frac{x_{e}^{2}}{1-x_{e}^{2}}\right) \operatorname{det}\left(\operatorname{Id}-P^{*}\right) .
\end{aligned}
$$

By Lemma 4.1, the 1.h.s. and the r.h.s. are both polynomials, so they are equal for all $\mathbf{x}$. Hence,

$$
Z_{\mathcal{L}}=\prod_{e \in E}\left(1-x_{e}^{2}\right)^{-1 / 2} \prod_{v \in V}\left(1+\sum_{e \ni v} \frac{x_{e}^{2}}{1-x_{e}^{2}}\right)^{-1 / 2} \operatorname{det}^{-\frac{1}{2}}\left(\operatorname{Id}-P^{*}\right)
$$

for all $\mathbf{x}$ such that $0<Z_{\mathcal{L}}<\infty$. Using the definition of $\lambda_{v}^{*}$ and the determinantal formula 4.3 concludes the proof.

Corollary 4.4. Let $\mathbf{x}$ be a vector of edge weights such that $\|\mathbf{x}\|_{\infty}<1$ and

$$
\sum_{e \ni v} \frac{x_{e}}{1+x_{e}} \leq 1 \quad \forall v \in V
$$

If $\exists v \in V$ such that

$$
\sum_{e \ni v} \frac{x_{e}}{1+x_{e}}<1
$$

then $Z_{\mathcal{L}}<\infty$ and Eq. (4.5) holds. If

$$
\sum_{e \ni v} \frac{x_{e}}{1+x_{e}}=1 \quad \forall v \in V
$$

then $Z_{\mathcal{L}}$ diverges.

In particular, in the homogeneous case, $x_{e} \equiv x$, on a $\delta$-regular graph, $Z_{\mathcal{L}}<\infty$ if $x<1 /(\delta-1)$ and $Z_{\mathcal{L}}$ diverges if $x=1 /(\delta-1)$.

Proof. It is easy to verify that, if $\sum_{e \ni v} \frac{x_{e}}{1+x_{e}} \leq 1$ for all $v \in V$ and, moreover, $\sum_{e \ni v} \frac{x_{e}}{1+x_{e}}<1$ for at least one $v \in V$, then $Z_{\mathcal{L}}<\infty$ and $\rho\left(P^{*}\right)<1$. If, in addition, $\|\mathbf{x}\|_{\infty}<1$, then Theorem 4.3 and the determinantal formula (4.3) hold. It is also easy to check that, if $\sum_{e \ni v} \frac{x_{e}}{1+x_{e}}=1$ for all $v \in V$, the transition matrix $P^{*}$ is Markovian and $Z_{\mathcal{L}}$ diverges.

Remark 4.5. A direct way of proving the homogeneous case result is by counting all possible non-backtracking walks on the graph, or by noticing that $\rho(\Lambda) \leq\|\Lambda\|_{1}=x(\delta-1)$ and using Lemma 4.1. Similarly, for any graph with vertices of degree $\operatorname{deg}_{v} \geq 2$, one can show that a sufficient condition to ensure that $Z_{\mathcal{L}}<\infty$ is: $x_{e}<1 /\left(\operatorname{deg}_{v}-1\right)$ for all $v \in V$ and all $e \ni v$.

We conclude this section with an interesting observation. As mentioned in the introduction, the partition function of the Gaussian free field in the form (4.3) can be expressed in terms of the partition function of a standard random walk loop soup, i.e., without the non-backtracking condition. (The proof of this fact is completely analogous to the proof of Lemma 4.1. The interested 
reader is referred to the proof of Lemma 1.2 of [7].) This provides a link between the partition function of the non-backtracking loop soup with transition probabilities given by $\Lambda$ and the random walk loop soup with transition probabilities given by $P^{*}$. It is possible that the connection between those two loop soups and their occupation fields is realized at a deeper level than that of the partition functions. Such a deeper connection, if it exists, could be revealed by an analysis of the determinantal formula for the Ihara zeta function given in Theorem 2 of [33].

\section{Exact Computations}

In this section, we will compute explicitly the free energy density of translation invariant models and the one-point function of homogeneous models on the torus $\mathbb{Z}^{d} /(n \mathbb{Z})^{d}$ for any $n \geq 1$, and $d \geq 1$, and hence after taking the thermodynamic limit, on all hypercubic lattices $\mathbb{Z}^{d}, d \geq 1$. We will also prove exponential decay of the two-point function in the subcritical regime. The reason why an exact solution is available is the fact that the partition function of the model is given by the square root of the determinant of a matrix (a situation similar to the one of the Ising and dimer model, and also the discrete Gaussian free field). It follows that the relevant quantities can be expressed in terms of the eigenvalues and can be computed for periodic graphs using the Fourier transform. Unlike in the Ising and dimer model case, the determinantal formulas are valid in all dimensions, as in the case of the discrete Gaussian free field.

\subsection{Partition Function and Free Energy Density}

Let $\mathcal{T}_{n}^{d}=\mathbb{Z}^{d} /(n \mathbb{Z})^{d}$ be a $d$-dimensional torus of size $n^{d}$. For a vertex $\mathbf{k}=$ $\left(k_{1}, k_{2}, \ldots, k_{d}\right) \in \mathcal{T}_{n}^{d}$, and a unit direction vector $\mathbf{v}=\left(v_{1}, v_{2}, \ldots, v_{d}\right)$ such that $v_{j}= \pm 1$ for some $j$, and $v_{i}=0$ for $i \neq j$, we will write $(\mathbf{k}, \mathbf{v})$ for the directed edge $\vec{e}$ with $t_{\vec{e}}=\mathbf{k}$ and $h_{\vec{e}}=\mathbf{k}+\mathbf{v}$. We consider a translation invariant weight vector $\mathbf{x}=\left(x_{1}, \ldots, x_{d}\right)$ that assigns weight $x_{j}$ to undirected versions of all edges of the form $(\mathbf{k}, \mathbf{v})$, where $\mathbf{v}$ is a unit vector in the $j$ th direction. In this case, the transition matrix (4.1) is given by

$$
\left[\Lambda_{n}^{d}\right]_{(\mathbf{k}, \mathbf{v}),(\mathbf{l}, \mathbf{u})}= \begin{cases}x_{j} & \text { if } \mathbf{k}+\mathbf{v}=\mathbf{l} \text { and } \mathbf{v} \neq-\mathbf{u} \\ 0 & \text { otherwise }\end{cases}
$$

Consider its Fourier transform

$$
\left[\hat{\Lambda}_{n}^{d}\right]_{(\mathbf{p}, \mathbf{v}),(\mathbf{q}, \mathbf{u})}=\frac{1}{n^{d}} \sum_{\mathbf{k}, \mathbf{l} \in \mathcal{T}_{n}^{d}} e^{-\frac{2 \pi i}{n}(\mathbf{p} \cdot \mathbf{k}-\mathbf{q} \cdot \mathbf{l})}\left[\Lambda_{n}^{d}\right]_{(\mathbf{k}, \mathbf{v}),(\mathbf{l}, \mathbf{u})} .
$$

It is easily seen that

$$
\left[\hat{\Lambda}_{n}^{d}\right]_{(\mathbf{p}, \mathbf{v}),(\mathbf{q}, \mathbf{u})}= \begin{cases}x_{j} e^{\frac{2 \pi i}{n} \mathbf{p} \cdot \mathbf{v}} & \text { if } \mathbf{p}=\mathbf{q} \text { and } \mathbf{v} \neq-\mathbf{u} \\ 0 & \text { otherwise }\end{cases}
$$


Note that $\hat{\Lambda}_{n}^{d}(\mathbf{p})$ is block diagonal with blocks indexed by the vertices $\mathbf{p} \in \mathcal{T}_{n}^{d}$ whose rows and columns correspond to the directed edges $\vec{e}$ satisfying $t_{\vec{e}}=\mathbf{p}$. Let $\hat{\Lambda}_{n}^{d}(\mathbf{p})$ denote the $2 d \times 2 d$ block corresponding to $\mathbf{p} \in \mathcal{T}_{n}^{d}$. Since $\hat{\Lambda}_{n}^{d}$ is similar to $\Lambda_{n}^{d}$, one has that

$$
\operatorname{det}\left(\operatorname{Id}-\Lambda_{n}^{d}\right)=\operatorname{det}\left(\operatorname{Id}-\hat{\Lambda}_{n}^{d}\right)=\prod_{\mathbf{p} \in \mathcal{T}_{n}^{d}} \operatorname{det}\left(\operatorname{Id}-\hat{\Lambda}_{n}^{d}(\mathbf{p})\right)
$$

where Id is the $2 d$-dimensional identity. One can explicitly compute these determinants as shown below.

Lemma 5.1. We have that

$$
\operatorname{det}\left(\operatorname{Id}-\hat{\Lambda}_{n}^{d}(\mathbf{p})\right)=\left[1+2 \sum_{i=1}^{d} \frac{x_{i}\left(x_{i}-\cos \left(\frac{2 \pi}{n} p_{i}\right)\right)}{1-x_{i}^{2}}\right] \prod_{i=1}^{d}\left(1-x_{i}^{2}\right)
$$

Proof. The proof is by induction on $d$. Let $z_{j}=e^{\frac{2 \pi i}{n} p_{j}}$. For $d=1$,

$$
\mathrm{Id}-\hat{\Lambda}_{n}^{1}(\mathbf{p})=\left(\begin{array}{cc}
1-x_{1} \bar{z}_{1} & 0 \\
0 & 1-x_{1} z_{1}
\end{array}\right)
$$

and the statement is true. Assume that it holds true for $d \leq k$, and consider the matrix $\Lambda_{n}^{k+1}(\mathbf{p})$ for $\mathbf{p} \in \mathcal{G}_{n}^{k+1}$. Let $\mathbf{p}^{\prime}=\left(p_{1}, p_{2}, \ldots, p_{k}\right) \in \mathcal{G}_{n}^{k}$ be the restriction of $\mathbf{p}$ to the first $k$ coordinates. For a number $a$, we will write $\underline{a}$ for a row or column vector with entries all equal to $a$. Let $M_{n}^{d}(\mathbf{p})$ be the matrix Id $-\hat{\Lambda}_{n}^{d}(\mathbf{p})$ where each row corresponding to a directed edge $(\mathbf{p}, \mathbf{v})$ is divided by $-x_{j} e^{\frac{2 \pi i}{n} \mathbf{p} \cdot \mathbf{v}}$, where $\mathbf{p} \cdot \mathbf{v}$ is either $p_{j}$ or $-p_{j}$. Note that $\operatorname{det}\left(\operatorname{Id}-\hat{\Lambda}_{n}^{d}(\mathbf{p})\right)=$ $\operatorname{det} M_{n}^{d}(\mathbf{p}) \prod_{i=1}^{d} x_{i}^{2}$. Using linearity of the determinant, we have

$$
\begin{aligned}
& \operatorname{det} M_{n}^{k+1}(\mathbf{p})=\left|\begin{array}{ccc}
1-\frac{z_{k+1}}{x_{k+1}} & 0 & \underline{1} \\
0 & 1-\frac{\bar{z}_{k+1}}{x_{k+1}} & \underline{1} \\
\underline{1} & \underline{1} & M_{n}^{k}\left(\mathbf{p}^{\prime}\right)
\end{array}\right| \\
& =\left|\begin{array}{ccc}
1-\frac{z_{k+1}}{x_{k+1}} & -1+\frac{\bar{z}_{k+1}}{x_{k+1}} & \underline{0} \\
0 & 1-\frac{\bar{z}_{k+1}}{x_{k+1}} & \underline{1} \\
\underline{1} & \underline{1} & M_{n}^{k}\left(\mathbf{p}^{\prime}\right)
\end{array}\right| \\
& =\left(1-\frac{z_{k+1}}{x_{k+1}}\right)\left|\begin{array}{cc}
1-\frac{\bar{z}_{k+1}}{x_{k+1}} & \underline{1} \\
\underline{1} & M_{n}^{k}\left(\mathbf{p}^{\prime}\right)
\end{array}\right|+\left(1-\frac{\bar{z}_{k+1}}{x_{k+1}}\right)\left|\begin{array}{ll}
0 & \underline{1} \\
\underline{1} & M_{n}^{k}\left(\mathbf{p}^{\prime}\right)
\end{array}\right| \\
& =\left(1-\frac{z_{k+1}}{x_{k+1}}\right)\left|\begin{array}{cc}
1 & \underline{1} \\
\underline{1} & M_{n}^{k}\left(\mathbf{p}^{\prime}\right)
\end{array}\right|+\left(1-\frac{z_{k+1}}{x_{k+1}}\right)\left|\begin{array}{cc}
-\frac{\bar{z}_{k+1}}{x_{k+1}} & \underline{0} \\
\underline{1} & M_{n}^{k}\left(\mathbf{p}^{\prime}\right)
\end{array}\right| \\
& +\left(1-\frac{\bar{z}_{k+1}}{x_{k+1}}\right)\left|\begin{array}{cc}
1 & \underline{1} \\
\underline{1} & M_{n}^{k}\left(\mathbf{p}^{\prime}\right)
\end{array}\right|+\left(1-\frac{\bar{z}_{k+1}}{x_{k+1}}\right)\left|\begin{array}{cc}
-1 & \underline{0} \\
\underline{1} & M_{n}^{k}\left(\mathbf{p}^{\prime}\right)
\end{array}\right| \\
& =\left(2-\frac{2}{x_{k+1}} \cos \left(\frac{2 \pi i}{n} p_{k+1}\right)\right)\left|\begin{array}{ll}
1 & \underline{1} \\
\underline{1} & M_{n}^{k}\left(\mathbf{p}^{\prime}\right)
\end{array}\right|+\left(\frac{1}{x_{k+1}^{2}}-1\right) \operatorname{det} M_{n}^{k}\left(\mathbf{p}^{\prime}\right) \text {, }
\end{aligned}
$$


Let $\bar{M}_{n}^{k}\left(\mathbf{p}^{\prime}\right)$ be the matrix $M_{n}^{k}\left(\mathbf{p}^{\prime}\right)$, where from each entry we subtract 1 . It is a block diagonal matrix with blocks of size 2 :

$$
\left(\begin{array}{cc}
-\frac{z_{i}}{x_{i}} & -1 \\
-1 & -\frac{\bar{z}_{i}}{x_{i}}
\end{array}\right)
$$

for $1 \leq i \leq k$, whose rows and columns correspond to the pairs of directed edges $\left(\mathbf{p}^{\prime}, \pm \mathbf{v}\right)$. Hence,

$$
\left|\begin{array}{cc}
1 & \underline{1} \\
1 & M_{n}^{k}\left(\mathbf{p}^{\prime}\right)
\end{array}\right|=\left|\begin{array}{ll}
1 & \underline{1} \\
\underline{0} & \bar{M}_{n}^{k}\left(\mathbf{p}^{\prime}\right)
\end{array}\right|=\operatorname{det} \bar{M}_{n}^{k}\left(\mathbf{p}^{\prime}\right)=\prod_{i=1}^{k}\left(\frac{1}{x_{i}^{2}}-1\right) .
$$

Therefore, by the induction assumption,

$$
\begin{aligned}
& \operatorname{det}\left(\operatorname{Id}-\hat{\Lambda}_{n}^{k+1}(\mathbf{p})\right) \\
& =2 x_{k+1}\left[x_{k+1}-\cos \left(\frac{2 \pi}{n} p_{k+1}\right)\right] \prod_{i=1}^{k}\left(1-x_{i}^{2}\right)+\left(1-x_{k+1}^{2}\right) \operatorname{det}\left(\operatorname{Id}-\hat{\Lambda}_{n}^{k}(\mathbf{p})\right) \\
& =\left[1+2 \sum_{i=1}^{k+1} \frac{x_{i}\left(x_{i}-\cos \left(\frac{2 \pi}{n} p_{i}\right)\right)}{1-x_{i}^{2}}\right] \prod_{i=1}^{k+1}\left(1-x_{i}^{2}\right) .
\end{aligned}
$$

From all these considerations, we obtain an exact formula for the partition function of the model on the torus.

Corollary 5.2. The partition function of the model on $\mathcal{T}_{n}^{d}$ with translation invariant weights $\mathbf{x}=\left(x_{1}, \ldots, x_{d}\right)$ satisfying

$$
\sum_{i=1}^{d} \frac{x_{i}}{1+x_{i}}<\frac{1}{2}
$$

is

$$
Z_{\mathcal{L}}=\prod_{\mathbf{p} \in \mathcal{T}_{n}^{d}}\left[1+2 \sum_{i=1}^{d} \frac{x_{i}\left(x_{i}-\cos \left(\frac{2 \pi}{n} p_{i}\right)\right)}{1-x_{i}^{2}}\right]^{-\frac{1}{2}} \prod_{i=1}^{d}\left(1-x_{i}^{2}\right)^{-\frac{n^{d}}{2}}
$$

Proof. We use (5.1) and Lemma 5.1, and note that the determinants of all blocks are positive whenever (5.3) holds true.

The free energy density of the model is defined as minus the logarithm of the partition function divided by the "volume" (the number of edges): ${ }^{3}$

$$
f(\mathbf{x})=-\frac{\log Z_{\mathcal{L}}}{|E|} .
$$

As an easy consequence of the corollary above, we obtain that the limiting free energy density as $\mathcal{T}_{n}^{d}$ approaches $\mathbb{Z}^{d}$ is given by an explicit formula.

\footnotetext{
${ }^{3}$ Since we will only compute the free energy density for tori and (hyper)cubic lattices and it will always be clear which case we are considering, we omit the graph from the notation.
} 
Corollary 5.3. The free energy density of the model on $\mathcal{T}_{n}^{d}$ with translation invariant weights $\mathbf{x}$ satisfying (5.3) in the thermodynamic limit $\mathcal{T}_{n}^{d} \nearrow \mathbb{Z}^{d}$ is given by

$$
f(\mathbf{x}) \cdot 2 d=\sum_{i=1}^{d} \log \left(1-x_{i}^{2}\right)+\frac{1}{(2 \pi)^{d}} \int_{[0,2 \pi]^{d}} \log \left[1+2 \sum_{i=1}^{d} \frac{x_{i}\left(x_{i}-\cos \alpha_{i}\right)}{1-x_{i}^{2}}\right] d \alpha .
$$

Note that the logarithm in the integral diverges as $\alpha \rightarrow 0$ on the critical surface

$$
\sum_{i=1}^{d} \frac{x_{i}}{1+x_{i}}=\frac{1}{2}
$$

From now on, we will simplify the setting by considering only homogenous models with a single parameter $x$ such that $\mathbf{x}=(x, \ldots, x)$. In this case, the critical point is $x_{c}=1 /(2 d-1)$. We now analyze the behavior of the singular part of the free energy density as $x \nearrow x_{c}$. We will write $A(x) \sim B(x)$ if $c_{1} B(x)+c_{2} \leq A(x) \leq C_{1} B(x)+C_{2}$, for some constants $c_{1}, c_{2}, C_{1}, C_{2}$ (depending on $d)$ as $x \nearrow x_{c}$.

Corollary 5.4. Let $\eta=d / 2$ if $d$ is even and $\eta=(d+1) / 2$ if $d$ is odd. If $n<\eta$, then $d^{n} f(x) / d x^{n}$ stays finite as $x \nearrow x_{c}$. If $d$ is even, then $d^{\eta} f(x) / d x^{\eta} \sim$ $\log \left(x_{c}-x\right)$, and if $d$ is odd, then $d^{\eta} f(x) / d x^{\eta} \sim\left(x_{c}-x\right)^{-1 / 2}$ as $x / x_{c}$.

Proof. Letting $2 x p(x)=(2 d-1) x^{2}-2 d x+1$ (so that $p\left(x_{c}\right)=0$ and $\frac{p(x)}{x_{c}-x} \rightarrow$ const $\neq 0$ as $x \nearrow x_{c}$ ), up to constants, one can write the singular part of $f$ as follows:

$$
\begin{aligned}
\int_{[0,2 \pi]^{d}} \log \left[p(x)+\sum_{i=1}^{d}\left(1-\cos \alpha_{i}\right)\right] \mathrm{d} \alpha \\
=2 \int_{[0, \pi]^{d}} \log \left[p(x)+\sum_{i=1}^{d}\left(1-\cos \alpha_{i}\right)\right] \mathrm{d} \alpha \\
\sim \int_{[0, \pi / 2]^{d}} \log \left[p(x)+\sum_{i=1}^{d}\left(1-\cos \alpha_{i}\right)\right] \mathrm{d} \alpha \\
=\int_{[0,1]^{d}} \log \left[p(x)+\sum_{i=1}^{d} y_{i}\right] \prod_{i=1}^{d} \frac{\mathrm{d} y_{i}}{\sqrt{y_{i}\left(2-y_{i}\right)}} \\
\sim \int_{[0,1]^{d}} \log \left[p(x)+\sum_{i=1}^{d} y_{i}\right] \prod_{i=1}^{d} y_{i}^{-1 / 2} \mathrm{~d} y_{i} \\
=2^{d} \int_{[0,1]^{d}} \log \left[p(x)+\sum_{i=1}^{d} z_{i}^{2}\right] \prod_{i=1}^{d} \mathrm{~d} z_{i} \\
\sim 2^{d} \int_{0}^{\sqrt{d}^{d}} \log \left[p(x)+r^{2}\right] r^{d-1} \mathrm{~d} r
\end{aligned}
$$




$$
=2^{d} d^{d / 2-1} \log [p(x)+d]-\frac{2^{d+2} x}{d} \int_{0}^{\sqrt{d}} \frac{r^{d+1}}{p(x)+r^{2}} \mathrm{~d} r .
$$

We see that the integral is convergent at $x=x_{c}$ for all $d \geq 2$. However, taking $n$ derivatives of $f$ generates a term containing the integral

$$
\int_{0}^{\sqrt{d}} \frac{r^{d-1} \mathrm{~d} r}{\left(p(x)+r^{2}\right)^{n}}
$$

At $x=x_{c}$, this integral is convergent if $2 n<d$ and divergent if $2 n \geq d$.

Writing $p=p(x)$, for $x$ sufficiently close to $x_{c}$, we have that

$$
\int_{0}^{\sqrt{d}} \frac{r^{d-1} \mathrm{~d} r}{\left(p+r^{2}\right)^{n}}=\int_{\sqrt{p}}^{\sqrt{d+p}} \frac{\left(s^{2}-p\right)^{\frac{d}{2}-1}}{s^{2 n-1}} \mathrm{~d} s \leq \int_{\sqrt{p}}^{\sqrt{d+p}} s^{d-1-2 n} \mathrm{~d} s
$$

and

$$
\begin{aligned}
\int_{0}^{\sqrt{d}} \frac{r^{d-1} \mathrm{~d} r}{\left(p+r^{2}\right)^{n}} & =\int_{0}^{\sqrt{p}} \frac{r^{d-1} \mathrm{~d} r}{\left(p+r^{2}\right)^{n}}+\int_{\sqrt{p}}^{\sqrt{d}} \frac{r^{d-1} \mathrm{~d} r}{\left(p+r^{2}\right)^{n}} \\
& \geq \frac{1}{(2 p)^{n}} \int_{0}^{\sqrt{p}} r^{d-1} \mathrm{~d} r+\frac{1}{2^{n}} \int_{\sqrt{p}}^{\sqrt{d}} r^{d-1-2 n} \mathrm{~d} r \\
& =\frac{1}{d 2^{n}} p^{d / 2-n}+\frac{1}{2^{n}} \int_{\sqrt{p}}^{\sqrt{d}} r^{d-1-2 n} \mathrm{~d} r
\end{aligned}
$$

The last statement of the lemma follows taking $n=\eta$.

\subsection{The One-Point Function}

In this section, we compute the one-point function of the homogenous model on $\mathcal{T}_{n}^{d}$ and $\mathbb{Z}^{d}$. We begin with a lemma which expresses it in terms of the Green's function. The result is proved by expressing the desired quantity in terms of a similar object in the soup of oriented loops, and then repeating a classic proof of an analogous statement for general loop soups [25]. Let

$$
G_{\vec{e}, \vec{g}}=\left[(\operatorname{Id}-\Lambda)^{-1}\right]_{\vec{e}, \vec{g}}
$$

be the Green's function for the non-backtracking random walk. If $X$ is a random variable, we will write $\langle X\rangle$ for its expectation.

Lemma 5.5. For any edge e,

$$
\left\langle N_{\mathcal{L}}(e)\right\rangle=G_{\vec{e}, \vec{e}}-1,
$$

where $\vec{e}$ is any of the two oriented versions of e. As a consequence,

$$
\left\langle\frac{1}{|E|} \sum_{e \in E} N_{\mathcal{L}}(e)\right\rangle=\frac{1}{2|E|} \operatorname{Tr}(\operatorname{Id}-\Lambda)^{-1}-1 .
$$

Proof. Let $\overrightarrow{\mathcal{L}}$ be the soup of unrooted but oriented loops with intensity $\frac{1}{2}$, i.e. a Poisson point process with intensity measure $\frac{1}{2} \vec{\mu}$, where $\vec{\mu}(\vec{\ell})=\mu(\ell)$, and 
where $\vec{\ell}$ is an oriented version of $\ell$. For an oriented edge $\vec{e}$, let $N_{\overrightarrow{\mathcal{L}}}(\vec{e})$ be the number of times $\overrightarrow{\mathcal{L}}$ visits $\vec{e}$. One has

$$
N_{\mathcal{L}}(e) \stackrel{d}{=} N_{\overrightarrow{\mathcal{L}}}(\vec{e})+N_{\overrightarrow{\mathcal{L}}}(-\vec{e}),
$$

and, since the distribution of $\overrightarrow{\mathcal{L}}$ is invariant under reversal of all loops,

$$
N_{\overrightarrow{\mathcal{L}}}(\vec{e}) \stackrel{d}{=} N_{\overrightarrow{\mathcal{L}}}(-\vec{e}) .
$$

Hence, $\left\langle N_{\mathcal{L}}(e)\right\rangle=2\left\langle N_{\overrightarrow{\mathcal{L}}}(\vec{e})\right\rangle$.

Fix an oriented edge $\vec{e}$. For $|t| \leq 1$, let

$$
\left[\Lambda_{t}\right]_{\vec{e}_{1}, \vec{e}_{2}}= \begin{cases}x_{e_{1}}\left(t \mathbb{1}_{\left\{\vec{e}_{1}=\vec{e}\right\}}+\mathbb{1}_{\left\{\vec{e}_{1} \neq \vec{e}\right\}}\right) & \text { if } h_{\vec{e}_{1}}=t_{\vec{e}_{2}} \text { and } t_{\vec{e}_{1}} \neq h_{\vec{e}_{2}}, \\ 0 & \text { otherwise, }\end{cases}
$$

and let $Z_{t}$ denote the partition function of the corresponding loop soup. Using expression (3.2) for the partition function and Lemma 4.1, we see that

$$
\begin{aligned}
\left\langle N_{\overrightarrow{\mathcal{L}}}(\vec{e})\right\rangle & =\left.\frac{\mathrm{d}}{\mathrm{d} t} \log Z_{t}\right|_{t=1} \\
& =\frac{\left.\frac{\mathrm{d}}{\mathrm{d} t} \operatorname{det}^{-\frac{1}{2}}\left(\operatorname{Id}-\Lambda_{t}\right)\right|_{t=1}}{\operatorname{det}^{-\frac{1}{2}}(\operatorname{Id}-\Lambda)} \\
& =-\frac{1}{2} \frac{\left.\frac{\mathrm{d}}{\mathrm{d} t} \operatorname{det}\left(\operatorname{Id}-\Lambda_{t}\right)\right|_{t=1}}{\operatorname{det}(\operatorname{Id}-\Lambda)} \\
& =\left.\frac{1}{2} \operatorname{Tr}\left[(\operatorname{Id}-\Lambda)^{-1} \frac{\mathrm{d}}{\mathrm{d} t} \Lambda_{t}\right]\right|_{t=1} \\
& =\frac{1}{2} \operatorname{Tr}\left[(\operatorname{Id}-\Lambda)^{-1} I_{\vec{e}} \Lambda\right] \\
& =\frac{1}{2} \operatorname{Tr}\left[I_{\vec{e}} \sum_{n=1}^{\infty} \Lambda^{n}\right] \\
& =\frac{1}{2} \operatorname{Tr}\left[I_{\vec{e}}\left((\operatorname{Id}-\Lambda)^{-1}-\mathrm{Id}\right)\right] \\
& =\frac{1}{2}\left(G_{\vec{e}, \vec{e}}-1\right),
\end{aligned}
$$

where the fourth identity follows from Jacobi's formula for the derivative of a determinant, and where $\left[I_{\vec{e}}\right] \vec{e}_{1}, \vec{e}_{2}=\mathbb{1}_{\left\{\vec{e}_{1}=\vec{e}_{2}=\vec{e}\right\}}$.

As in the case of the partition function, using the above result which relates the one-point function to the underlying matrix, exact computations can be made for the homogenous model.

Lemma 5.6. The eigenvalues of $\hat{\Lambda}_{n}^{d}(\mathbf{p})$ are $\pm x$ with multiplicity $d-1$, and

$$
x \frac{2 d-1}{a_{\mathbf{p}} \pm \sqrt{a_{\mathbf{p}}^{2}-2 d+1}}
$$

with multiplicity 1 , where $a_{\mathbf{p}}=\sum_{i=1}^{d} \cos \left(\frac{2 \pi}{n} p_{i}\right)$.

Proof. The result follows from Lemma 5.1. 
Note that it follows that the spectral radius of $\Lambda_{n}^{d}$ is equal to $(2 d-1) x$ and is achieved by one of the multiplicity-one eigenvalues of $\hat{\Lambda}_{n}^{d}(\mathbf{p})$ for $\mathbf{p}=$ $(0, \ldots, 0)$.

Corollary 5.7. For any edge e of $\mathcal{T}_{n}^{d}$,

$$
\left\langle N_{\mathcal{L}}(e)\right\rangle=\frac{1}{d n^{d}} \sum_{\mathbf{p} \in \mathcal{T}_{n}^{d}} \frac{1-x \sum_{i=1}^{d} \cos \left(\frac{2 \pi}{n} p_{i}\right)}{1+(2 d-1) x^{2}-2 x \sum_{i=1}^{d} \cos \left(\frac{2 \pi}{n} p_{i}\right)}+A(x),
$$

and hence, in the thermodynamic limit,

$$
\lim _{\mathcal{T}_{n}^{d} \nearrow \mathbb{Z}^{d}}\left\langle N_{\mathcal{L}}(e)\right\rangle=\frac{1}{d(2 \pi)^{d}} \int_{[0,2 \pi]^{d}} \frac{1-x \sum_{i=1}^{d} \cos \alpha_{i}}{1+(2 d-1) x^{2}-2 x \sum_{i=1}^{d} \cos \alpha_{i}} \mathrm{~d} \alpha+A(x),
$$

where $A(x)=\frac{d-1}{d}\left(1-x^{2}\right)^{-1}-1$ is smooth on $(0,1)$.

Proof. Let $\sigma_{n}^{d}(\mathbf{p})$ be the spectrum of $\hat{\Lambda}_{n}^{d}(\mathbf{p})$. Using Lemmas 5.5 and 5.6, we have

$$
\begin{aligned}
\left\langle N_{\mathcal{L}}(e)\right\rangle+1 & =\left\langle\frac{1}{|E|} \sum_{e^{\prime} \in E} N_{\mathcal{L}}\left(e^{\prime}\right)\right\rangle+1 \\
& =\frac{1}{2|E|} \operatorname{Tr}\left(\mathrm{Id}-\Lambda_{n}^{d}\right)^{-1} \\
& =\frac{1}{2 \mathrm{~d} n^{d}} \sum_{\mathbf{p} \in \mathcal{T}_{n}^{d}} \sum_{\lambda \in \sigma_{n}^{d}(\mathbf{p})}(1-\lambda)^{-1} \\
& =\frac{d-1}{d}\left(1-x^{2}\right)^{-1}+\frac{1}{\mathrm{~d} n^{d}} \sum_{\mathbf{p} \in \mathcal{T}_{n}^{d}} \frac{1-x a_{\mathbf{p}}}{1+(2 d-1) x^{2}-2 x a_{\mathbf{p}}} .
\end{aligned}
$$

Corollary 5.8. As $x \nearrow x_{c}=1 /(2 d-1), \lim _{\mathcal{T}_{n}^{d} \nearrow \mathbb{Z}^{d}}\left\langle N_{\mathcal{L}}(e)\right\rangle$ stays bounded for $d \geq 3$, and diverges logarithmically for $d=2$.

Proof. Corollary 5.7 and a computation analogous to the one in the proof of Corollary 5.4 yield

$$
\lim _{\mathcal{T}_{n}^{d} \nearrow \mathbb{Z}^{d}}\left\langle N_{\mathcal{L}}(e)\right\rangle \sim 2^{d} \int_{0}^{\sqrt{d}}\left[p(x)+r^{2}\right]^{-1} r^{d-1} \mathrm{~d} r .
$$

The integral is convergent for all $d \geq 3$; for $d=2$, one has

$$
4 \int_{0}^{\sqrt{2}} \frac{r \mathrm{~d} r}{\left[p(x)+r^{2}\right]}=2 \log \left(\frac{p(x)+2}{p(x)}\right)
$$

which diverges logarithmically as $x \nearrow x_{c}$.

\section{The Distribution of $N_{\mathcal{L}}(e)$}

In this section, we compute the probability generating function of $N_{\mathcal{L}}(e)$ and then use the result to prove a limit theorem for the two-dimensional edgeoccupation field. 
For $|z| \leq 1$, let

$$
p_{e}(z)=\sum_{n=0}^{\infty} \mathbb{P}\left(N_{\mathcal{L}}(e)=n\right) z^{n}
$$

be the probability generating function of $N_{\mathcal{L}}(e)$. For a directed edge $\vec{e}$, let $F_{\vec{e}}$ be the partition function of directed loops $\vec{\omega}$ rooted at $\vec{e}$ which do not visit $-\vec{e}$ and such that $\vec{\omega}_{i}=\vec{e}$ only for $i=1$ and $i=|\vec{\omega}|+1$ (that is, the sum over all such loops of the weights of the loops). Let $F_{\vec{e}}^{\prime}$ be the partition function of walks $\vec{\omega}$ rooted at $\vec{e}$ such that $\vec{\omega}_{i}=\vec{e}$ only for $i=1$ and $\vec{\omega}_{i}=-\vec{e}$ only for $i=|\vec{\omega}|+1$.

\section{Lemma 6.1.}

$$
p_{e}(z)=\left(\frac{1-z F_{-\vec{e}}-z^{2} F_{\vec{e}}^{\prime} F_{-\vec{e}}^{\prime}}{1-F_{-\vec{e}}-F_{\vec{e}}^{\prime} F_{-\vec{e}}^{\prime}}\right)^{-1 / 2}
$$

Proof. Let $Z_{z}$ be the partition function of the soup of oriented loops with intensity measure $\frac{1}{2} \vec{\mu}_{z}$, where $\vec{\mu}_{z}(\vec{\ell})=\mu(\ell) z^{N_{\ell}(e)}$. One has

$$
p_{e}(z)=\frac{Z_{z}}{Z_{1}}=\exp \left(\frac{1}{2} \sum_{\vec{\ell}: e \in \vec{\ell}} \vec{\mu}_{z}(\vec{\ell})\right) / \exp \left(\frac{1}{2} \sum_{\vec{\ell}: e \in \vec{\ell}} \vec{\mu}_{1}(\vec{\ell})\right) .
$$

Using the identity

$$
\sum_{\vec{\ell}: e \in \vec{\ell}} \vec{\mu}_{z}(\vec{\ell})=\sum_{\vec{\ell}: \vec{e} \in \vec{\ell}} \vec{\mu}_{z}(\vec{\ell})+\sum_{\vec{\ell}:-\vec{e} \in \vec{\ell}, \vec{e} \notin \vec{\ell}} \vec{\mu}_{z}(\vec{\ell})
$$

and a well-known fact (see, for instance, Lemma 9.3.2 in [20] or Lemma 4 in [26] for a proof in the non-backtracking case), one gets

$$
\exp \left(\frac{1}{2} \sum_{\vec{\ell}: e \in \vec{\ell}} \vec{\mu}_{z}(\vec{\ell})\right)=\left(1-\tilde{G}_{\vec{e}, \vec{e}}^{z}\right)^{-1 / 2}\left(1-z F_{-\vec{e}}\right)^{-1 / 2},
$$

where $\tilde{G}_{\vec{e}, \vec{e}}^{z}$ is the partition function of oriented loops $\vec{\omega}$ rooted at $\vec{e}$ such that $\vec{\omega}_{i}=\vec{e}$ only for $i=1$ and $i=|\vec{\omega}|+1$, weighted with the weight $x^{z}(\vec{\omega})=$ $x(\vec{\omega}) z^{N_{\vec{\omega}}(e)}$. Splitting these loops according to their first and last visit to $-\vec{e}$, one has

$$
\tilde{G}_{\vec{e}, \vec{e}}^{z}=z F_{\vec{e}}^{\prime}\left(1-z F_{-\vec{e}}\right)^{-1} z F_{-\vec{e}}^{\prime},
$$

which together with (6.1) finishes the proof.

Contrary to dimension three and higher, in two dimensions the edgeoccupation field is not defined at the critical point (see, for example, Corollary 5.8). Nevertheless, in $\mathcal{T}_{n}^{2}$ for any $n$, including $n=\infty$, one can use Lemma 6.1 and the next corollary to prove a limit theorem, as $x \nearrow 1 / 3$, for the field normalized by its expectation. 


\section{Corollary 6.2.}

$$
\left\langle N_{\mathcal{L}}(e)\right\rangle=\frac{1}{2} \frac{F_{-\vec{e}}+2 F_{\vec{e}}^{\prime} F_{-\vec{e}}^{\prime}}{1-F_{-\vec{e}}-F_{\vec{e}}^{\prime} F_{-\vec{e}}^{\prime}}
$$

Proof. We use the fact that $\left\langle N_{\mathcal{L}}(e)\right\rangle=\left.\frac{d}{d z} p_{e}(z)\right|_{z=1}$ and Lemma 6.1.

Theorem 6.3. Fix $n$ (possibly $n=\infty$ ) and consider the loop soup $\mathcal{L}$ in $\mathcal{T}_{n}^{2}$. Then, for any edge $e$, as $x \nearrow 1 / 3, N_{\mathcal{L}}(e) /\left\langle N_{\mathcal{L}}(e)\right\rangle$ converges in distribution to the square of the standard normal distribution.

Proof. We will use Lévy's continuity theorem. Let $\varphi(t)$ be the characteristic function of $N_{\mathcal{L}}(e) /\left\langle N_{\mathcal{L}}(e)\right\rangle$, and let $\varepsilon=1-F_{\vec{e}}-F_{\vec{e}}^{\prime} F_{-\vec{e}}^{\prime}$ and $C=F_{-\vec{e}}+2 F_{\vec{e}}^{\prime} F_{-\vec{e}}^{\prime}$. From Corollaries 5.7 and 6.2, we can deduce that, as $x \nearrow 1 / 3, \varepsilon \rightarrow 0$ and $F_{-\vec{e}}$, $F_{\vec{e}}^{\prime}$ and $F_{-\vec{e}}^{\prime}$ remain bounded. Hence, by Lemma 6.1 and Corollary 6.2,

$$
\begin{aligned}
\lim _{x \nearrow 1 / 3} \varphi(t) & =\lim _{\varepsilon \rightarrow 0}\left(1-\frac{\left(e^{2 i t \varepsilon / C}-1\right) F_{-\vec{e}}+\left(e^{4 i t \varepsilon / C}-1\right) F_{\vec{e}}^{\prime} F_{-\vec{e}}^{\prime}}{\varepsilon}\right)^{-1 / 2} \\
& =(1-2 i t)^{-1 / 2},
\end{aligned}
$$

which is the characteristic function of the square of the standard normal distribution.

\section{The Two-Point Function}

In this section, we show the existence of a subcritical regime with exponential decay of correlations. We let

$$
\bar{N}_{\mathcal{L}}(e)=N_{\mathcal{L}}(e)-\left\langle N_{\mathcal{L}}(e)\right\rangle
$$

be the truncated two-point function. We denote by $N_{\ell}(e)$ the number of visits of the loop $\ell$ to $e \in E$, and write $e \in \ell$ if $\ell$ visits $e$ at least once.

Lemma 7.1. For any pair of edges e, g, we have that

$$
\left\langle\bar{N}_{\mathcal{L}}(e) \bar{N}_{\mathcal{L}}(g)\right\rangle=\sum_{\ell: e, g \in \ell} N_{\ell}(e) N_{\ell}(g) \mu(\ell) .
$$

Proof. We will use the obvious identity

$$
N_{\mathcal{L}}(e)=\sum_{\ell: e \in \ell, g \notin \ell}(\# \ell) N_{\ell}(e)+\sum_{\ell: e, g \in \ell}(\# \ell) N_{\ell}(e),
$$

where $\# \ell$ is the multiplicity of $\ell$ in $\mathcal{L}$. Using the fact that $\mathcal{L}$ is a Poisson point process, this gives

$$
\begin{aligned}
\left\langle\bar{N}_{\mathcal{L}}(e) \bar{N}_{\mathcal{L}}(g)\right\rangle & =\left\langle N_{\mathcal{L}}(e) N_{\mathcal{L}}(g)\right\rangle-\left\langle N_{\mathcal{L}}(e)\right\rangle\left\langle N_{\mathcal{L}}(g)\right\rangle \\
& =\left\langle\left(\sum_{\ell: e, g \in \ell}(\# \ell) N_{\ell}(e)\right)\left(\sum_{\ell: e, g \in \ell}(\# \ell) N_{\ell}(g)\right)\right\rangle
\end{aligned}
$$




$$
\begin{aligned}
& -\left\langle\sum_{\ell: e, g \in \ell}(\# \ell) N_{\ell}(e)\right\rangle\left\langle\sum_{\ell: e, g \in \ell}(\# \ell) N_{\ell}(g)\right\rangle \\
= & \sum_{\ell, \ell^{\prime}: e, g \in \ell, \ell^{\prime}} N_{\ell}(e) N_{\ell^{\prime}}(g)\left\langle(\# \ell)\left(\# \ell^{\prime}\right)\right\rangle \\
- & \sum_{\ell, \ell^{\prime}: e, g \in \ell, \ell^{\prime}} N_{\ell}(e) N_{\ell^{\prime}}(g)\langle \# \ell\rangle\left\langle \# \ell^{\prime}\right\rangle \\
= & \sum_{\ell: e, g \in \ell} N_{\ell}(e) N_{\ell}(g)\left(\left\langle(\# \ell)^{2}\right\rangle-\langle \# \ell\rangle^{2}\right) \\
= & \sum_{\ell: e, g \in \ell} N_{\ell}(e) N_{\ell}(g) \mu(\ell) .
\end{aligned}
$$

On a regular lattice where each vertex has $2 d$ nearest neighbors, the number of rooted, non-backtracking walks of length $k$ is bounded above by $(2 d-1)^{k}$. Lemma 7.1 then makes it clear that for $x<1 /(2 d-1)$ one should expect exponential decay of the truncated two-point function, identifying $x<$ $1 /(2 d-1)$ as the subcritical regime and $x=1 /(2 d-1)$ as the critical point.

We now express the truncated two-point function in terms of the Green's function, and use the expression to give a proof of exponential decay in the subcritical regime.

Lemma 7.2. For any pair of edges $e, g$, we have that

$$
2\left\langle\bar{N}_{\mathcal{L}}(e) \bar{N}_{\mathcal{L}}(g)\right\rangle=G_{\vec{e}, \vec{g}} G_{\vec{g}, \vec{e}}+G_{-\vec{e}, \vec{g}} G_{\vec{g},-\vec{e}}+G_{-\vec{e},-\vec{g}} G_{-\vec{g},-\vec{e}}+G_{\vec{e},-\vec{g}} G_{-\vec{g}, \vec{e}} .
$$

Proof. As for the one-point function, we consider the soup of oriented loops $\overrightarrow{\mathcal{L}}$. We have

$$
\left\langle N_{\mathcal{L}}(e) N_{\mathcal{L}}(g)\right\rangle=\left\langle\left(N_{\overrightarrow{\mathcal{L}}}(\vec{e})+N_{\overrightarrow{\mathcal{L}}}(-\vec{e})\right)\left(N_{\overrightarrow{\mathcal{L}}}(\vec{g})+N_{\overrightarrow{\mathcal{L}}}(-\vec{g})\right)\right\rangle .
$$

Let us compute $\left\langle N_{\overrightarrow{\mathcal{L}}}(\vec{e}) N_{\overrightarrow{\mathcal{L}}}(\vec{g})\right\rangle$. For $|s|,|t| \leq 1$, let $Z_{s, t}$ be the partition function of the soup of oriented loops with intensity measure $\frac{1}{2} \vec{\mu}_{s, t}$, where

$$
\vec{\mu}_{s, t}(\vec{\ell})=\vec{\mu}(\vec{\ell}) s^{N_{\vec{\ell}}(\vec{e})} t^{N_{\vec{\ell}}(\vec{g})},
$$

with $N_{\vec{\ell}}(\vec{e})$ being the number of visits of $\vec{\ell}$ to $\vec{e}$. Using the form of the partition function (see (3.2)), one has

$$
\begin{aligned}
\left\langle N_{\overrightarrow{\mathcal{L}}}(\vec{e}) N_{\overrightarrow{\mathcal{L}}}(\vec{g})\right\rangle & =\frac{\left.\frac{\partial}{\partial s} \frac{\partial}{\partial t} Z_{s, t}\right|_{s, t=1}}{Z_{1,1}} \\
& =\frac{\left.\frac{\partial}{\partial s} \frac{\partial}{\partial t} \exp \left(\log Z_{s, t}\right)\right|_{s=t=1}}{Z_{1,1}} \\
& =\left.\frac{\partial}{\partial s} \frac{\partial}{\partial t} \log Z_{s, t}\right|_{s, t=1}+\left.\frac{\partial}{\partial s} \log Z_{s, t} \frac{\partial}{\partial t} \log Z_{s, t}\right|_{s, t=1} \\
& =\left.\frac{1}{2} \frac{\partial}{\partial s} G_{\vec{e}, \vec{e}}^{s}\right|_{s=1}+\frac{1}{4}\left\langle N_{\mathcal{L}}(e)\right\rangle\left\langle N_{\mathcal{L}}(g)\right\rangle,
\end{aligned}
$$


where we used Lemma 5.5, and where $G^{s}$ is the Green's function for the nonbacktracking walk with weight $x_{s}(\vec{\omega})=x(\vec{\omega}) s^{N_{\vec{\omega}}(\vec{g})}$. Let $\tilde{G}_{\vec{g}, \vec{g}}$ be the partition function of oriented loops $\vec{\omega}$ rooted at $\vec{g}$ such that $\vec{\omega}_{i}=\vec{g}$ only for $i=1$ and $i=|\vec{\omega}|+1$ (that is, the sum over all such loops of the weights of the loops). Splitting loops that visit $\vec{g}$ multiple times into loops that visit $\vec{g}$ only once, one can see that $G_{\vec{g}, \vec{g}}^{s}=\left(1-s \tilde{G}_{\vec{g}, \vec{g}}\right)^{-1}-1$ and, hence, $G_{\vec{e}, \vec{e}}^{s}=C s\left(1-s \tilde{G}_{\vec{g}, \vec{g}}\right)^{-1}$, where $C$ does not depend on $s$ (the additional factor $s$ comes from the first visit of the walk to $\vec{g}$ ). Note that only loops visiting $\vec{g}$ survive the differentiation $\frac{\partial}{\partial s}$, and that

$$
\left.\frac{\partial}{\partial s} s\left(1-s \tilde{G}_{\vec{g}, \vec{g}}\right)^{-1}\right|_{s=1}=\left(1-\tilde{G}_{\vec{g}, \vec{g}}\right)^{-2}=G_{\vec{g}, \vec{g}}^{2} .
$$

Decomposing the rooted loops starting at $\vec{e}$ according to their first and last visits to $\vec{g}$ and using the identity above we obtain that $\left.\frac{\partial}{\partial s} G_{\vec{e}, \vec{e}}^{s}\right|_{s=1}=G_{\vec{e}, \vec{g}} G_{\vec{g}, \vec{e}}$, which finishes the proof.

Corollary 7.3 (Exponential decay). For the homogeneous model on the torus $\mathcal{T}_{n}^{d}$ with $x_{e}=x<1 /(2 d-1)$, one has

$$
\left\langle\bar{N}_{\mathcal{L}}(e) \bar{N}_{\mathcal{L}}(g)\right\rangle \leq 2 \frac{(x(2 d-1))^{2 d(e, g)}}{(1-x(2 d-1))^{2}},
$$

where $d(e, g)$ is the graph distance between e and $g$.

Proof. By Lemma 7.2, it is enough to prove that $G_{\vec{e}, \vec{g}} \leq \frac{(x(2 d-1))^{d(e, g)}}{1-x(2 d-1)}$. The induced $L^{1}$ norm of the transition matrix is $\left\|\Lambda_{n}^{d}\right\|_{1}=x(2 d-1)$. Hence,

$$
\begin{aligned}
G_{\vec{e}, \vec{g}} & =\left[\left(\mathrm{Id}-\Lambda_{n}^{d}\right)^{-1}\right]_{\vec{e}, \vec{g}} \\
& =\left[\left(\Lambda_{n}^{d}\right)^{d(e, g)}\left(\mathrm{Id}-\Lambda_{n}^{d}\right)^{-1}\right]_{\vec{e}, \vec{g}} \\
& \leq\left\|\left(\Lambda_{n}^{d}\right)^{d(e, g)}\left(\mathrm{Id}-\Lambda_{n}^{d}\right)^{-1}\right\|_{1} \\
& \leq\left\|\Lambda_{n}^{d}\right\|_{1}^{d(e, g)}\left(1-\left\|\Lambda_{n}^{d}\right\|_{1}\right)^{-1} .
\end{aligned}
$$

\section{A Reflection Positive Spin Model}

In this section, we consider the loop soup on the square lattice, defined as the graph with vertices $\mathbb{Z}^{2}$ and edges between nearest neighbor vertices. The dual graph $\left(\mathbb{Z}^{2}\right)^{*}$ is again a square lattice. To each vertex $v^{*}$ of the dual graph $\left(\mathbb{Z}^{2}\right)^{*}$, we associate a $( \pm 1)$-valued $(\operatorname{spin})$ variable $\sigma_{v^{*}}$.

We define a spin model on the vertices of $\left(\mathbb{Z}^{2}\right)^{*}$ by taking a loop soup $\mathcal{L}$ in $\mathbb{Z}^{2}$ and assigning, to each dual vertex $v^{*}$, spin

$$
\sigma_{v^{*}}=\exp \left(\frac{i}{2} \sum_{\ell \in \mathcal{L}} \theta_{\ell}\left(v^{*}\right)\right),
$$

where $\theta_{\ell}\left(v^{*}\right)$ is the winding angle (a multiple of $\pm 2 \pi$ ) of loop $\ell$ around $v^{*}$ (and $i$ here denotes the imaginary unit). Here, we assume that there are only finitely many loops surrounding any dual vertex. This is true for any $x<1 / 3$. 
We will now show that this spin model is reflection positive. (See [6] for more information on the concept and use of reflection positivity in the context of lattice spin models. The question of reflection positivity in the loop soup context is addressed in Chapter 9 of [25].) $\mathbb{Z}^{2}$ has a natural reflection symmetry along any line $l$ going through a set of dual vertices. Such a line splits $\mathbb{Z}^{2}$ into two halves, $\mathbb{Z}_{+}^{2}$ and $\mathbb{Z}_{-}^{2}$. We also split accordingly the dual graph $\left(\mathbb{Z}^{2}\right)^{*}$ into two halves, $\left(\mathbb{Z}_{+}^{2}\right)^{*}$ and $\left(\mathbb{Z}_{-}^{2}\right)^{*}$, such that $\left(\mathbb{Z}_{+}^{2}\right)^{*} \cap\left(\mathbb{Z}_{-}^{2}\right)^{*}=V_{l}^{*}$, where $V_{l}^{*}$ is the set of vertices of $\left(\mathbb{Z}^{2}\right)^{*}$ that lie on $l$.

Let $\mathcal{F}^{+}$(respectively, $\mathcal{F}^{-}$) denote the set of all functions of the spin variables $\left(\sigma_{v^{*}}\right)_{v^{*} \in\left(\mathbb{Z}_{+}^{2}\right)^{*}}$ (respectively, $\left.\left(\sigma_{v^{*}}\right)_{v^{*} \in\left(\mathbb{Z}_{-}^{2}\right)^{*}}\right)$. Let $\vartheta$ denote the reflection operator, $\vartheta: \mathcal{F}^{ \pm} \rightarrow \mathcal{F}^{\mp}$, whose action on spins is given by $\vartheta\left(\sigma_{v^{*}}\right)=\sigma_{\vartheta\left(v^{*}\right)}$, and let $\mathbb{E}$ denote expectation with respect to the loop soup.

Lemma 8.1 (Reflection positivity) For all functions $f, g \in \mathcal{F}^{+}, \mathbb{E}(f \vartheta g)=$ $\mathbb{E}(g \vartheta f)$ and $\mathbb{E}(f \vartheta f) \geq 0$.

Proof. Take $v^{*} \in\left(\mathbb{Z}_{ \pm}^{2}\right)^{*}$ and draw a path in $\left(\mathbb{Z}_{ \pm}^{2}\right)^{*}$ from $v^{*}$ to infinity. Let $n_{v^{*}}$ be the number of loops and arcs of the soup in $\mathbb{Z}_{ \pm}^{2}$ which cross the path an odd number of times. One can see that $\sigma_{v^{*}}=(-1)^{n_{v^{*}}}$, and hence the spin model in $\left(\mathbb{Z}_{ \pm}^{2}\right)^{*}$ is a function of the loops and arcs of the soup contained $\mathbb{Z}_{ \pm}^{2}$.

Let $E_{l}$ denote the edges of $\mathbb{Z}^{2}$ crossed by the reflection line $l$. Theorem 3.3 implies, therefore, that $f$ and $\vartheta g$ are independent conditional on $\left.N_{S}\right|_{E_{l}}$. Using reflection symmetry, this gives $\mathbb{E}\left(f \vartheta g\left|N_{S}\right|_{E_{l}}\right)=\mathbb{E}\left(f\left|N_{S}\right|_{E_{l}}\right) \mathbb{E}\left(\vartheta g\left|N_{S}\right|_{E_{l}}\right)$. The proof of the lemma follows immediately from the law of total expectation.

Defining the spin model on the square lattice is convenient to express reflection positivity, but one can of course define the same model on other twodimensional graphs. Consider, for example, the spin model defined via (8.1) on a finite subset of the square or hexagonal lattice with zero boundary condition $\xi$ and equal edge weights $x_{e}=x \forall e \in E$. We define the (unnormalized) magnetization field as $\sum_{v^{*}} \sigma_{v^{*}} \delta_{v^{*}}$, where $\delta_{v^{*}}$ denotes the Dirac delta at $v^{*}$. We conjecture that, if $x=1 / 3$ in the case of the square lattice or $x=1 / 2$ in the case of the hexagonal lattice, the magnetization field, when properly rescaled, has a continuum scaling limit which gives rise to one of the conformal fields discussed in $[9,32]$, namely the winding field with $\lambda=1 / 2$ and $\beta=\pi$ in the language of [9]. (Note that, if $x<1 / 3$ on the square lattice or $x<1 / 2$ on the hexagonal lattice, the truncated two-point function can be shown to decay exponentially by arguments similar to those in the proof of Lemma 7.1.)

Some evidence in favor of this conjecture comes from [4]. In that paper, the authors consider the scaling limit of a quantity that can be interpreted as the one-point function of a spin model defined via (8.1), but using walks without the non-backtracking condition. They express the limit in terms of the Brownian loop soup [21] in a way that is consistent with our conjecture. We expect that the non-backtracking condition does not change the scaling limit of the loop soup, so the same computation should apply to the one-point function of our spin model. 
Our conjecture and Lemma 8.1 suggest that the winding field of $[9,32]$ with $\lambda=1 / 2$ and $\beta=\pi$ is reflection positive and satisfies the OsterwalderSchrader axioms of Euclidean field theory [27].

We also conjecture that, both in the case of the square lattice with $x=$ $1 / 3$ and in the case of the hexagonal lattice with $x=1 / 2$, in the continuum scaling limit, the collection of boundaries of the spin clusters converges to $\mathrm{CLE}_{4}$, the Conformal Loop Ensemble with parameter $\kappa=4$.

\section{Acknowledgements}

The first author acknowledges the support of Vidi Grant 639.032.916 of the Netherlands Organization for Scientific Research (NWO). He also thanks Roberto Fernandez and Chuck Newman for useful suggestions. The second author was partially supported by the Knut and Alice Wallenberg Foundation. This research was conducted while the second author was at Brown University and Chalmers University of Technology. The second author also thanks VU University Amsterdam for its hospitality during two visits while the paper was completed. Both authors are grateful to Yves Le Jan for several useful conversations and remarks on a previous draft of the paper, and for pointing their attention to references [23,24], and to the Ihara zeta function. They also thank Wendelin Werner for a useful discussion on the spatial Markov property of loop soups, and two anonymous referees for their detailed comments and useful suggestions.

Open Access. This article is distributed under the terms of the Creative Commons Attribution 4.0 International License (http://creativecommons.org/licenses/ by/4.0/), which permits unrestricted use, distribution, and reproduction in any medium, provided you give appropriate credit to the original author(s) and the source, provide a link to the Creative Commons license, and indicate if changes were made.

\section{References}

[1] Aizenman, M.: Proof of the triviality of $\phi_{d}{ }^{4}$ Field theory and some mean-field features of Ising models for $d>4$. Phys. Rev. Lett. 47, 1-4 (1981)

[2] Aizenman, M.: Geometric analysis of $\phi^{4}$ fields and Ising models. Parts I and II. Commun. Math. Phys. 86(1), 1-48 (1982)

[3] Aizenman, M., Duminil-Copin, H., Sidoravicius, V.: Random Currents and Continuity of Ising Model's Spontaneous Magnetization. Commun. Math. Phys. 334(2), 719-742 (2014)

[4] Beneš, C., Viklund, F. J., Lawler, G. F.: Scaling limit of the loop-erased random walk Green's function. (2014). arXiv:1402.7345v1 [math.PR]

[5] Berlin, T.H., Kac, M.: The spherical model of a ferromagnet. Phys. Rev. 86, 821-835 (1952)

[6] Biskup, M.: Reflection positivity and phase transitions in lattice spin models. In: Methods of Contemporary Mathematical Statistical Physics, vol. 1970. Lecture Notes in Mathematics, pp. 1-86. Springer-Verlag, Berlin (2009) 
[7] Brydges, D., Fröhlich, J., Spencer, T.: The random walk representation of classical spin systems and correlation inequalities. Commun. Math. Phys. 83(1), 123-150 (1982)

[8] Brydges, D., Fröhlich, J., Sokal, A.D.: The random-walk representation of classical spin systems and correlation inequalities. II. The skeleton inequalities. Commun. Math. Phys. 91(1), 117-139 (1983)

[9] Camia, F., Gandolfi, A., Kleban, M.: Conformal correlation functions in the Brownian loop soup. Nucl. Phys. B 902, 483-507 (2016)

[10] Crane, L.: 2-d physics and 3-d topology. Commun. Math. Phys. 135(3), 615-640 (1991)

[11] Crane, L.: Conformal field theory, spin geometry, and quantum gravity. Phys. Lett. B 259(3), 243-248 (1991)

[12] Fitzner, R., van der Hofstad, R.: Non-backtracking random walk. J. Stat. Phys. 150(2), 264-284 (2013)

[13] Fröhlich, J.: On the triviality of $\lambda \phi_{d}^{4}$ theories and the approach to the critical point in $d_{(-)}>4$ dimensions. Nucl. Phys. B 200(2), 281-296 (1982)

[14] Hashimoto, K.-I.: Zeta functions of finite graphs and representations of p-adic groups. Automorphic forms and geometry of arithmetic varieties, pp. 211-280 (1989)

[15] Horton, M.D., Stark, H.M., Terras, A.A.: What are zeta functions of graphs and what are they good for? Contemp. Math. 415, 173-190 (2006)

[16] Ihara, Y.: On discrete subgroups of the two by two projective linear group over p-adic fields. J. Math. Soc. Japan 18(3), 219-235 (1966)

[17] Kac, M., Clive Ward, J.: A combinatorial solution of the two-dimensional Ising model. Phys. Rev. 88(6), 1332-1337 (1952)

[18] Kager, W., Lis, M., Meester, R.: The signed loop approach to the Ising model: Foundations and critical point. J. Stat. Phys. 152(2), 353-387 (2013)

[19] Lawler, G. F., Trujillo Ferreras, J. A.: Random walk loop soup. Trans. Am. Math. Soc. 359(2), 767-787 (2007) (electronic)

[20] Lawler, G.F., Limic, V.: Random walk: a modern introduction. volume 123 of Cambridge Studies in Advanced Mathematics. Cambridge University Press, Cambridge (2010)

[21] Lawler, G.F., Werner, W.: The Brownian loop soup. Probab. Theory Relat. Fields 128(4), 565-588 (2004)

[22] Le Jan, Y.: Markov loops and renormalization. Ann. Probab. 38(3), 1280-1319 (2010)

[23] Le Jan, Y.: Markov loops, coverings and fields. (2016). arXiv:1602.02708

[24] Le Jan, Y.: Markov loops, free field and Eulerian networks. J. Math. Soc. Japan 67(4), 1671-1680, 10 (2015)

[25] Le Jan, Y.: Lectures from the 38th Probability Summer School held in SaintFlour, 2008. In: Markov paths, loops and fields. Lecture Notes in Mathematics. École d'Été de Probabilités de Saint-Flour. [Saint-Flour Probability Summer School], vol. 2026. Springer, Heidelberg (2011). ISBN: 978-3-642-21215-4

[26] Lis, M.: A short proof of the Kac-Ward formula. Ann. Inst. Henri Poincaré Comb. Phys. Interact 3, 45-53 (2016) 
[27] Osterwalder, K., Schrader, R.: Axioms for Euclidean Green's functions. Commun. Math. Phys. 31(2), 83-112 (1973)

[28] Penrose, R.: Angular momentum: an approach to combinatorial space-time. In Quantum theory and beyond. Cambridge University Press, Cambridge (1971)

[29] Rovelli, C., Smolin, L.: Spin networks and quantum gravity. Phys. Rev. D 53, 5743-5759 (1995)

[30] Stark, H.M., Terras, A.A.: Zeta functions of finite graphs and coverings. Adv. Math. 121(1), 124-165 (1996)

[31] Symanzik, K.: Euclidean quantum field theory. Local Quantum Theory. Academic, New York (1969)

[32] van de Brug, T., Camia, F., Lis, M.: Conformal fields from Brownian loops (2017) (In preparation)

[33] Watanabe, Y., Fukumizu, K.: Graph zeta function in the bethe free energy and loopy belief propagation. In: Bengio, Y., Schuurmans, D., Lafferty, J.D., Williams, C.K.I., Culotta, A., (eds.) Advances in Neural Information Processing Systems 22, pp. 2017-2025. Curran Associates, Inc. (2009)

[34] Werner, W.: On the spatial Markov property of soups of unoriented and oriented loops. In: Sém. Probab. XLVIII. Springer (2016) (To appear)

Federico Camia

New York University Abu Dhabi

Saadiyat Island

Abu Dhabi

United Arab Emirates

e-mail: federico.camia@nyu.edu

and

Department of Mathematics

VU University Amsterdam

De Boelelaan 1081a

1081 HV Amsterdam

The Netherlands

Marcin Lis

Statistical Laboratory Centre for Mathematical Sciences

Cambridge University

Wilberforce Road

Cambridge CB3 0WB

UK

e-mail: m.lis@statslab.cam.ac.uk

Communicated by Abdelmalek Abdesselam.

Received: February 23, 2016.

Accepted: September 10, 2016. 